\title{
Supersymmetric economical 3-3-1 model
}

\author{
P.V. Dong ${ }^{\mathrm{a}}$, D.T. Huong ${ }^{\mathrm{a}}$, M.C. Rodriguez ${ }^{\mathrm{b}}$, H.N. Long ${ }^{\mathrm{a}, *}$ \\ ${ }^{a}$ Institute of Physics, VAST, PO Box 429, Bo Ho, Hanoi 10000, Vietnam \\ ${ }^{\mathrm{b}}$ Fundação Universidade Federal do Rio Grande-FURG, Departamento de Física, Av. Itália, $\mathrm{km} 8$, \\ Campus Carreiros, 96201-900 Rio Grande, RS, Brazil
}

Received 11 August 2006; received in revised form 19 January 2007; accepted 8 March 2007

Available online 13 March 2007

\begin{abstract}
The supersymmetric extension of the economical 3-3-1 model is presented. The constraint equations and the gauge boson identification establish a relation between the vacuum expectation values (VEVs) at the top and bottom elements of the Higgs triplet $\chi$ and its supersymmetric counterpart $\chi^{\prime}$. Because of this relation, the exact diagonalization of neutral gauge boson sector has been performed. The gauge bosons and their associated Goldstone ones mix in the same way as in non-supersymmetric version. This is also correct in the case of gauginos. The eigenvalues and eigenstates in the Higgs sector are derived. The model contains a heavy neutral Higgs boson with mass equal to those of the neutral non-Hermitian gauge boson $X^{0}$ and a charged scalar with mass equal to those of the $W$ boson in the Standard Model, i.e., $m_{\varrho_{1}}=m_{W}$. This result is in good agreement with the present estimation: $m_{H^{ \pm}}>79.3 \mathrm{GeV}, \mathrm{CL}=95 \%$. We also show that the boson sector and the fermion sector gain masses in the same way as in the non-supersymmetric case. (C) 2007 Elsevier B.V. All rights reserved.
\end{abstract}

\section{PACS: 12.60.Jv; 12.60.Fr; 14.80.Ly}

Keywords: Supersymmetric models; Extensions of electroweak Higgs sector; Supersymmetric partners of known particles

\section{Introduction}

Recent neutrino experimental results [1-3] establish the fact that neutrinos have masses and the Standard Model (SM) must be extended. The generation of neutrino masses is an important

\footnotetext{
* Corresponding author.

E-mail addresses: pvdong@iop.vast.ac.vn (P.V. Dong), dthuong@iop.vast.ac.vn (D.T. Huong), mcrodriguez@einstein.fisica.furg.br (M.C. Rodriguez), hnlong@iop.vast.ac.vn (H.N. Long).
} 
issue in any realistic extension of the SM. In general, the values of these masses (of the order of, or less than, $1 \mathrm{eV}$ ) that are needed to explain all neutrino oscillation data are not enough to put strong constraints on model building. It means that several models can induce neutrino masses and mixing compatible with experimental data. So, instead of proposing models built just to explain the neutrino properties, it is more useful to consider what are the neutrino masses that are predicted in any particular model which has motivation other than the explanation of neutrino physics.

The SM is exceedingly successful in describing leptons, quarks and their interactions. Nevertheless, the SM is not considered as the ultimate theory since neither the fundamental parameters, masses and couplings, nor the symmetry pattern are predicted. These elements are merely built into the model. Likewise, the spontaneous electroweak symmetry breaking is simply parametrized by a single Higgs doublet field.

Even though many aspects of the SM are experimentally supported to a very accuracy, the embedding of the model into a more general framework is to be expected. The argument is closely connected to the mechanism of the electroweak symmetry breaking. If the Higgs boson is light, the SM can naturally be embedded in a grand unified theory, the so-called GUT. The large energy gap between the low electroweak scale and the high GUT scale can be stabilized by supersymmetry. Supersymmetry actually provides the link between the experimentally explored interactions at electroweak energy scales and physics at scales close to the Planck scale $M_{\mathrm{pl}} \approx 10^{19} \mathrm{GeV}$ where gravity is important.

On the other hand, the possibility of a gauge symmetry based on the following symmetry $\mathrm{SU}(3)_{C} \otimes \mathrm{SU}(3)_{L} \otimes \mathrm{U}(1)_{X}(3-3-1)$ [4-6] is particularly interesting, because it explains some fundamental questions that are eluded in the SM. The main motivations to study this kind of model are:

(1) The family number must be multiple of three;

(2) It explains why $\sin ^{2} \theta_{W}<\frac{1}{4}$ is observed;

(3) It solves the strong $\mathrm{CP}$ problem;

(4) It is the simplest model that includes bileptons of both types: scalar and vectors ones;

(5) The model has several sources of CP violation.

In one of the 3-3-1 models [6] which is anomaly free, the particle content is given by ${ }^{1}$

$$
\begin{aligned}
& L_{a L}=\left(v_{a}, l_{a}, v_{a}^{c}\right)_{L}^{T} \sim(\mathbf{3},-1 / 3), \quad l_{a R} \sim(\mathbf{1},-1), \quad a=1,2,3, \\
& Q_{1 L}=\left(u_{1}, d_{1}, u^{\prime}\right)_{L}^{T} \sim(\mathbf{3}, 1 / 3), \quad Q_{\alpha L}=\left(d_{\alpha},-u_{\alpha}, d_{\alpha}^{\prime}\right)_{L}^{T} \sim\left(\mathbf{3}^{*}, 0\right), \quad \alpha=2,3, \\
& u_{i R} \sim(\mathbf{1}, 2 / 3), \quad d_{i R} \sim(\mathbf{1},-1 / 3), \quad i=1,2,3, \\
& u_{R}^{\prime} \sim(\mathbf{1}, 2 / 3), \quad d_{\alpha R}^{\prime} \sim(\mathbf{1},-1 / 3),
\end{aligned}
$$

where the values in the parentheses denote quantum numbers based on the $\left(\mathrm{SU}(3)_{L}, \mathrm{U}(1)_{X}\right)$ symmetry. The exotic quarks $u^{\prime}$ and $d_{\alpha}^{\prime}$ in this case take the same electric charges as of the usual quarks, i.e., $q_{u^{\prime}}=2 / 3, q_{d_{\alpha}^{\prime}}=-1 / 3$. The spontaneous symmetry breaking in this model is achieved by two Higgs scalar triplets only

$$
\chi=\left(\chi_{1}^{0}, \chi^{-}, \chi_{2}^{0}\right)^{T} \sim(\mathbf{3},-1 / 3), \quad \rho=\left(\rho_{1}^{+}, \rho^{0}, \rho_{2}^{+}\right)^{T} \sim(\mathbf{3}, 2 / 3)
$$

\footnotetext{
1 In this article the notation is slightly different from those in Ref. [7].
} 
with all the neutral components $\chi_{1}^{0}, \chi_{2}^{0}$ and $\rho^{0}$ developing the vacuum expectation values.

Such a scalar sector is minimal, and therefore it has been called the economical 3-3-1 model $[8,9]$. In a series of papers, we have developed and proved that this non-supersymmetric version is consistent, realistic and very rich in physics. Let us remind some steps in the development: The general Higgs sector is very simple (economical) and consists of three physical scalars (two neutral and one charged) and eight Goldstone bosons-the needed number for massive gauge ones [10]. In Refs. [11,12], we have shown that the model under the consideration is realistic, by the mean that, at the one-loop level, all fermions gain consistent masses. In addition, the model contains a majoron associated with $\chi_{1}^{0}$ responsible for the Majorana masses of neutrinos.

Supersymmetry (SUSY) that transforms fermions into bosons and vice versa is a leading candidate for physics beyond the SM [13]. The existence of such a non-trivial extension is highly constrained by theoretical principles. One of the motivations for supersymmetry is that it can help to understand the hierarchy problem. The supersymmetric version of the 3-3-1 model with righthanded neutrinos [6] has already been constructed in Refs. [7,14]. The neutrino masses in this case were studied in Ref. [15], and the proton instability was considered in Ref. [16].

It was shown that [17], the 3-3-1 models are the first gauge ones containing the candidates for self-interaction dark matter (SIDM) [18] with the condition given by Spergel and Steinhardt [19]. It was shown that [10] the economical 3-3-1 model does not furnish any candidate for SIDM. This directly relates to the scalar sector in which a significant number of fields and couplings is reduced (to compare, see Ref. [14]). With the larger field content, the supersymmetric version of the model is expected to provide candidates for dark matter, particularly for the SIDM.

The aim of this work is to study the supersymmetric version of the economical 3-3-1 model.

The outline of this paper is as follows. In Section 2 we present a fermion and scalar content in the supersymmetric economical 3-3-1 model. The supersymmetric Lagrangian and breaking are given in Section 3. In Sections 4, 5 and 6 we deal with the gauge boson, fermion and Higgs sectors of the model. Finally, we summarize our results and make conclusions in Section 7.

\section{Particle content of supersymmetric economical 3-3-1 model}

To proceed further, the necessary features of the supersymmetric economical 3-3-1 model [9] will be presented. The superfield content in this paper is defined in a standard way as follows

$$
\hat{F}=(\tilde{F}, F), \quad \hat{S}=(S, \tilde{S}), \quad \hat{V}=(\lambda, V),
$$

where the components $F, S$ and $V$ stand for the fermion, scalar and vector fields of the economical 3-3-1 model while their superpartners are denoted as $\tilde{F}, \tilde{S}$ and $\lambda$, respectively $[7,13]$.

The superfields for the leptons under the 3-3-1 gauge group transform as

$$
\hat{L}_{a L}=\left(\hat{v}_{a}, \hat{l}_{a}, \hat{v}_{a}^{c}\right)_{L}^{T} \sim(\mathbf{1}, \mathbf{3},-1 / 3), \quad \hat{l}_{a L}^{c} \sim(\mathbf{1}, \mathbf{1}, 1),
$$

where $\hat{v}_{L}^{c}=\left(\hat{v}_{R}\right)^{c}$ and $a=1,2,3$ is a generation index.

It is worth mentioning that, in the economical version the first generation of quarks should be different from others [11]. The superfields for the left-handed quarks of the first generation are in triplets

$$
\hat{Q}_{1 L}=\left(\hat{u}_{1}, \hat{d}_{1}, \hat{u}^{\prime}\right)_{L}^{T} \sim(\mathbf{3}, \mathbf{3}, 1 / 3),
$$

where the right-handed singlet counterparts are given by

$$
\hat{u}_{1 L}^{c}, \hat{u}_{L}^{\prime c} \sim\left(\mathbf{3}^{*}, \mathbf{1},-2 / 3\right), \quad \hat{d}_{1 L}^{c} \sim\left(\mathbf{3}^{*}, \mathbf{1}, 1 / 3\right) .
$$


Conversely, the superfields for the last two generations transform as antitriplets

$$
\hat{Q}_{\alpha L}=\left(\hat{d}_{\alpha},-\hat{u}_{\alpha}, \hat{d}_{\alpha}^{\prime}\right)_{L}^{T} \sim\left(\mathbf{3}, \mathbf{3}^{*}, 0\right), \quad \alpha=2,3,
$$

where the right-handed counterparts are in singlets

$$
\hat{u}_{\alpha L}^{c} \sim\left(\mathbf{3}^{*}, \mathbf{1},-2 / 3\right), \quad \hat{d}_{\alpha L}^{c}, \hat{d}_{\alpha L}^{\prime c} \sim\left(\mathbf{3}^{*}, \mathbf{1}, 1 / 3\right) .
$$

The primes superscript on usual quark types ( $u^{\prime}$ with the electric charge $q_{u^{\prime}}=2 / 3$ and $d^{\prime}$ with $q_{d^{\prime}}=-1 / 3$ ) indicate that those quarks are exotic ones. The mentioned fermion content, which belongs to that of the 3-3-1 model with right-handed neutrinos [6,9] is, of course, free from anomaly.

The two superfields $\hat{\chi}$ and $\hat{\rho}$ are at least introduced to span the scalar sector of the economical 3-3-1 model [10]:

$$
\begin{aligned}
& \hat{\chi}=\left(\hat{\chi}_{1}^{0}, \hat{\chi}^{-}, \hat{\chi}_{2}^{0}\right)^{T} \sim(1,3,-1 / 3), \\
& \hat{\rho}=\left(\hat{\rho}_{1}^{+}, \hat{\rho}^{0}, \hat{\rho}_{2}^{+}\right)^{T} \sim(1,3,2 / 3) .
\end{aligned}
$$

To cancel the chiral anomalies of higgsino sector, the two extra superfields $\hat{\chi}^{\prime}$ and $\hat{\rho}^{\prime}$ must be added as follows

$$
\begin{aligned}
& \hat{\chi}^{\prime}=\left(\hat{\chi}_{1}^{\prime 0}, \hat{\chi}^{\prime+}, \hat{\chi}_{2}^{\prime 0}\right)^{T} \sim\left(1,3^{*}, 1 / 3\right), \\
& \hat{\rho}^{\prime}=\left(\hat{\rho}_{1}^{\prime-}, \hat{\rho}^{\prime 0}, \hat{\rho}_{2}^{\prime-}\right)^{T} \sim\left(1,3^{*},-2 / 3\right) .
\end{aligned}
$$

In this model, the $\mathrm{SU}(3)_{L} \otimes \mathrm{U}(1)_{X}$ gauge group is broken via two steps:

$$
\mathrm{SU}(3)_{L} \otimes \mathrm{U}(1)_{X} \stackrel{w, w^{\prime}}{\longrightarrow} \mathrm{SU}(2)_{L} \otimes \mathrm{U}(1)_{Y} \stackrel{v, v^{\prime}, u, u^{\prime}}{\longrightarrow} \mathrm{U}(1)_{Q},
$$

where the VEVs are defined by

$$
\begin{array}{ll}
\sqrt{2}\langle\chi\rangle^{T}=(u, 0, w), & \sqrt{2}\left\langle\chi^{\prime}\right\rangle^{T}=\left(u^{\prime}, 0, w^{\prime}\right), \\
\sqrt{2}\langle\rho\rangle^{T}=(0, v, 0), & \sqrt{2}\left\langle\rho^{\prime}\right\rangle^{T}=\left(0, v^{\prime}, 0\right) .
\end{array}
$$

The VEVs $w$ and $w^{\prime}$ are responsible for the first step of the symmetry breaking while $u, u^{\prime}$ and $v$, $v^{\prime}$ are for the second one. Therefore, they have to satisfy the constraints:

$$
u, u^{\prime}, v, v^{\prime} \ll w, w^{\prime} .
$$

The vector superfields $\hat{V}_{c}, \hat{V}$ and $\hat{V}^{\prime}$ containing the usual gauge bosons are, respectively, associated with the $\mathrm{SU}(3)_{C}, \mathrm{SU}(3)_{L}$ and $\mathrm{U}(1)_{X}$ group factors. The colour and flavour vector superfields have expansions in the Gell-Mann matrix bases $T^{a}=\lambda^{a} / 2(a=1,2, \ldots, 8)$ as follows

$$
\hat{V}_{c}=\frac{1}{2} \lambda^{a} \hat{V}_{c a}, \quad \hat{\bar{V}}_{c}=-\frac{1}{2} \lambda^{a *} \hat{V}_{c a}, \quad \hat{V}=\frac{1}{2} \lambda^{a} \hat{V}_{a}, \quad \hat{\bar{V}}=-\frac{1}{2} \lambda^{a *} \hat{V}_{a},
$$

where an overbar ${ }^{-}$indicates complex conjugation. For the vector superfield associated with $\mathrm{U}(1)_{X}$, we normalize as follows

$$
X \hat{V}^{\prime}=\left(X T^{9}\right) \hat{B}, \quad T^{9} \equiv \frac{1}{\sqrt{6}} \operatorname{diag}(1,1,1) .
$$

In the following, we are denoting the gluons by $g^{a}$ and their respective gluino partners by $\lambda_{c}^{a}$, with $a=1, \ldots, 8$. In the electroweak sector, $V^{a}$ and $B$ stand for the $\mathrm{SU}(3)_{L}$ and $\mathrm{U}(1)_{X}$ gauge bosons with their gaugino partners $\lambda_{V}^{a}$ and $\lambda_{B}$, respectively. 


\section{Lagrangian}

With the superfields given above, we can now construct the supersymmetric economical 3-3-1 model containing the Lagrangians: $\mathcal{L}_{\text {susy }}+\mathcal{L}_{\text {soft }}$, where the first term is supersymmetric part, whereas the last term breaks explicitly the supersymmetry.

\subsection{Supersymmetric Lagrangian}

The supersymmetric Lagrangian can be decomposed into four relevant parts

$$
\mathcal{L}_{\text {susy }}=\mathcal{L}_{\text {gauge }}+\mathcal{L}_{\text {lepton }}+\mathcal{L}_{\text {quark }}+\mathcal{L}_{\text {scalar }}
$$

The first term has the form

$$
\begin{aligned}
\mathcal{L}_{\text {gauge }}= & \frac{1}{4} \int d^{2} \theta \mathcal{W}_{c a} \mathcal{W}_{c a}+\frac{1}{4} \int d^{2} \theta \mathcal{W}_{a} \mathcal{W}_{a}+\frac{1}{4} \int d^{2} \theta \mathcal{W}^{\prime} \mathcal{W}^{\prime} \\
& +\frac{1}{4} \int d^{2} \bar{\theta} \overline{\mathcal{W}}_{c a} \overline{\mathcal{W}}_{c a}+\frac{1}{4} \int d^{2} \bar{\theta} \overline{\mathcal{W}}_{a} \overline{\mathcal{W}}_{a}+\frac{1}{4} \int d^{2} \bar{\theta} \overline{\mathcal{W}}^{\prime} \overline{\mathcal{W}}^{\prime},
\end{aligned}
$$

where the chiral superfields $\mathcal{W}_{c}, \mathcal{W}$ and $\mathcal{W}^{\prime}$ are defined by

$$
\begin{aligned}
& \mathcal{W}_{c \zeta}=-\frac{1}{8 g_{s}} \bar{D} \bar{D} e^{-2 g_{s} \hat{V}_{c}} D_{\zeta} e^{2 g_{s} \hat{V}_{c}}, \quad \mathcal{W}_{\zeta}=-\frac{1}{8 g} \bar{D} \bar{D} e^{-2 g \hat{V}} D_{\zeta} e^{2 g \hat{V}}, \\
& \mathcal{W}_{\zeta}^{\prime}=-\frac{1}{4} \bar{D} \bar{D} D_{\zeta} \hat{V}^{\prime}, \quad \zeta=1,2,
\end{aligned}
$$

with the gauge couplings $g_{s}, g$ and $g^{\prime}$ respective to $\mathrm{SU}(3)_{C}, \mathrm{SU}(3)_{L}$ and $\mathrm{U}(1)_{X}$. The $D_{\zeta}$ and $\bar{D}_{\dot{\zeta}}$ are the chiral covariant derivatives of SUSY algebra as presented in [13].

The second and third terms are given by

$$
\mathcal{L}_{\text {lepton }}=\int d^{4} \theta\left[\hat{\bar{L}}_{a L} e^{2\left(g \hat{V}-\frac{g^{\prime}}{3} \hat{V}^{\prime}\right)} \hat{L}_{a L}+\hat{\bar{l}}_{a L}^{c} e^{2 g^{\prime} \hat{V}^{\prime}} \hat{l}_{a L}^{c}\right],
$$

and

$$
\begin{aligned}
\mathcal{L}_{\text {quark }}= & \int d^{4} \theta\left[\hat{\bar{Q}}_{1 L} e^{2\left(g_{s} \hat{V}_{c}+g \hat{V}+\frac{g^{\prime}}{3} \hat{V}^{\prime}\right)} \hat{Q}_{1 L}+\hat{\bar{Q}}_{\alpha L} e^{2\left(g_{s} \hat{V}_{c}+g \hat{\bar{V}}\right)} \hat{Q}_{\alpha L}\right. \\
& +\hat{\bar{u}}_{i L}^{c} e^{2\left(g_{s} \hat{\bar{V}}_{c}-\frac{2 g^{\prime}}{3} \hat{V}^{\prime}\right)} \hat{u}_{i L}^{c}+\hat{\bar{d}}_{i L}^{c} e^{2\left(g_{s} \hat{\bar{V}}_{c}+\frac{g^{\prime}}{3} \hat{V}^{\prime}\right)} \hat{d}_{i L}^{c} \\
& \left.+\hat{\bar{u}}_{L}^{c} e^{2\left(g_{s} \hat{\bar{V}}_{c}-\frac{2 g^{\prime}}{3} \hat{V}^{\prime}\right)} \hat{u}_{L}^{\prime c}+\hat{\overline{\vec{d}}}_{\alpha L}^{\prime c} e^{2\left(g_{s} \hat{\bar{V}}_{c}+\frac{g^{\prime}}{3} \hat{V}^{\prime}\right)} \hat{d}_{\alpha L}^{\prime c}\right] .
\end{aligned}
$$

Finally, the last term can be written as

$$
\begin{aligned}
\mathcal{L}_{\text {scalar }}= & \int d^{4} \theta\left\{\hat{\bar{\chi}} e^{2\left[g \hat{V}+g^{\prime}\left(-\frac{1}{3}\right) \hat{V}^{\prime}\right]} \hat{\chi}+\hat{\bar{\rho}} e^{2\left[g \hat{V}+g^{\prime}\left(\frac{2}{3}\right) \hat{V}^{\prime}\right]} \hat{\rho}+\hat{\bar{\chi}}^{\prime} e^{2\left[g \hat{\bar{V}}+g^{\prime}\left(\frac{1}{3}\right) \hat{V}^{\prime}\right]} \hat{\chi}^{\prime}\right. \\
& \left.+\hat{\bar{\rho}}^{\prime} e^{2\left[g \hat{\bar{V}}+g^{\prime}\left(-\frac{2}{3}\right) \hat{V}^{\prime}\right]} \hat{\rho}^{\prime}\right\}+\left(\int d^{2} \theta W+\text { H.c. }\right)
\end{aligned}
$$

with

$$
W=\frac{W_{2}}{2}+\frac{W_{3}}{3},
$$


where

$$
W_{2}=\mu_{0 a} \hat{L}_{a L} \hat{\chi}^{\prime}+\mu_{\chi} \hat{\chi} \hat{\chi}^{\prime}+\mu_{\rho} \hat{\rho} \hat{\rho}^{\prime}
$$

and

$$
\begin{aligned}
W_{3}= & \lambda_{1 a b} \hat{L}_{a L} \hat{\rho}^{\prime} \hat{l}_{b L}^{c}+\lambda_{2 a} \epsilon \hat{L}_{a L} \hat{\chi} \hat{\rho}+\lambda_{3 a b} \epsilon \hat{L}_{a L} \hat{L}_{b L} \hat{\rho}+\kappa_{1 i} \hat{Q}_{1 L} \hat{\chi}^{\prime} \hat{u}_{i L}^{c} \\
& +\kappa_{1}^{\prime} \hat{Q}_{1 L} \hat{\chi}^{\prime} \hat{u}_{L}^{\prime c}+\kappa_{2 i} \hat{Q}_{1 L} \hat{\rho}^{\prime} \hat{d}_{i L}^{c}+\kappa_{2 \alpha}^{\prime} \hat{Q}_{1 L} \hat{\rho}^{\prime} \hat{d}_{\alpha L}^{\prime c}+\kappa_{3 \alpha i} \hat{Q}_{\alpha L} \hat{\rho} \hat{u}_{i L}^{c} \\
& +\kappa_{3 \alpha}^{\prime} \hat{Q}_{\alpha L} \hat{\rho} \hat{u}_{L}^{\prime c}+\kappa_{4 \alpha i} \hat{Q}_{\alpha L} \hat{\chi} \hat{d}_{i L}^{c}+\kappa_{4 \alpha \beta}^{\prime} \hat{Q}_{\alpha L} \hat{\chi} \hat{d}_{\beta L}^{\prime c}+\epsilon f_{1 \alpha \beta \gamma} \hat{Q}_{\alpha L} \hat{Q}_{\beta L} \hat{Q}_{\gamma L} \\
& +\xi_{1 i \beta j} \hat{d}_{i L}^{c} \hat{d}_{\beta L}^{\prime c} \hat{u}_{j L}^{c}+\xi_{2 i \beta} \hat{d}_{i L}^{c} \hat{d}_{\beta L}^{\prime c} \hat{u}_{L}^{\prime c}+\xi_{3 i j k} \hat{d}_{i L}^{c} \hat{d}_{j L}^{c} \hat{u}_{k L}^{c}+\xi_{4 i j} \hat{d}_{i L}^{c} \hat{d}_{j L}^{c} \hat{u}_{L}^{\prime c} \\
& +\xi_{5 \alpha \beta i} \hat{d}_{\alpha L}^{\prime c} \hat{d}_{\beta L}^{\prime c} \hat{u}_{i L}^{c}+\xi_{6 \alpha \beta} \hat{d}_{\alpha L}^{\prime c} \hat{d}_{\beta L}^{\prime c} \hat{u}_{L}^{c}+\xi_{7 a \alpha j} \hat{L}_{a L} \hat{Q}_{\alpha L} \hat{d}_{j L}^{c}+\xi_{8 a \alpha \beta} \hat{L}_{a L} \hat{Q}_{\alpha L} \hat{d}_{\beta L}^{\prime c} .
\end{aligned}
$$

The coefficients $\mu_{0 a}, \mu_{\rho}$ and $\mu_{\chi}$ have mass dimension, while all coefficients in $W_{3}$ are dimensionless.

To find interactions contained in the supersymmetric Lagrangian, we first obtain all the kinetic terms [13]:

$$
\begin{aligned}
\mathcal{L}_{\text {kinetic }}= & \left(D^{\mu} \chi\right)^{+} D_{\mu} \chi+\left(D^{\mu} \rho\right)^{+} D_{\mu} \rho+\left(\bar{D}^{\mu} \tilde{Q}_{\alpha L}\right)^{+} \bar{D}_{\mu} \tilde{Q}_{\alpha L} \\
& +\left(\bar{D}^{\mu} \chi^{\prime}\right)^{+} \bar{D}_{\mu} \chi^{\prime}+\left(\bar{D}^{\mu} \rho^{\prime}\right)^{+} \bar{D}_{\mu} \rho^{\prime}+\left(D^{\mu} \tilde{Q}_{1 L}\right)^{+} D_{\mu} \tilde{Q}_{1 L} \\
& +\left(D^{\mu} \tilde{L}_{a L}\right)^{+} D_{\mu} \tilde{L}_{a L}+\left(D_{1}^{\mu} \tilde{d}_{i L}^{c}\right)^{+} D_{1 \mu} \tilde{d}_{i L}^{c}+\left(D_{1}^{\mu} \tilde{u}_{i L}^{c}\right)^{+} D_{1 \mu} \tilde{u}_{i L}^{c} \\
& +\left(D_{1}^{\mu} \tilde{u}_{L}^{\prime c}\right)^{+} D_{1 \mu} \tilde{u}_{L}^{\prime c}+\left(D_{1}^{\mu} \tilde{d}_{\alpha L}^{\prime c}\right)^{+} D_{1 \mu} \tilde{d}_{\alpha L}^{\prime c}+i \bar{L}_{a L} \bar{\sigma}^{\mu} D_{\mu} L_{a L} \\
& +i \bar{Q}_{1 L} \bar{\sigma}^{\mu} D_{\mu} Q_{1 L}+i \bar{Q}_{\alpha L} \bar{\sigma}^{\mu} \bar{D}_{\mu} Q_{\alpha L}+i \bar{l}_{a L}^{c} \bar{\sigma}^{\mu} D_{1 \mu} l_{a L}^{c} \\
& +i \bar{u}_{i L}^{c} \bar{\sigma}^{\mu} D_{1 \mu} u_{i L}^{c}+i \bar{d}_{i L}^{c} \bar{\sigma}^{\mu} D_{1 \mu} d_{i L}^{c}+i \bar{u}_{L}^{\prime c} \bar{\sigma}^{\mu} D_{1 \mu} u_{L}^{\prime c}+i \bar{d}_{\alpha L}^{\prime c} \bar{\sigma}^{\mu} D_{1 \mu} d_{\alpha L}^{c c} \\
& -\frac{1}{4} F_{c a}^{\mu \nu} F_{c a \mu \nu}-\frac{1}{4} F_{a}^{\mu \nu} F_{a \mu \nu}-\frac{1}{4} F^{\mu \nu} F_{\mu \nu}+\mathcal{L}_{\text {gaugino }}+\mathcal{L}_{\text {Higgsinos }},
\end{aligned}
$$

where

$$
\begin{aligned}
& \mathcal{L}_{\text {gaugino }}=i \bar{\lambda}_{c}^{a} \bar{\sigma}^{\mu} D_{\mu}^{c} \lambda_{c}^{a}+i \bar{\lambda}_{V}^{a} \bar{\sigma}^{\mu} D_{\mu}^{L} \lambda_{V}^{a}+i \bar{\lambda}_{B} \bar{\sigma}^{\mu} \partial_{\mu} \lambda_{B}, \\
& \mathcal{L}_{\text {Higgsinos }}=i \overline{\tilde{\rho}} \bar{\sigma}^{\mu} D_{\mu} \tilde{\rho}+i \overline{\tilde{\chi}} \bar{\sigma}^{\mu} D_{\mu} \tilde{\chi}+i \overline{\tilde{\rho}}^{\prime} \bar{\sigma}^{\mu} \bar{D}_{\mu} \tilde{\rho}^{\prime}+i \overline{\tilde{\chi}}^{\prime} \bar{\sigma}^{\mu} \bar{D}_{\mu} \tilde{\chi}^{\prime} .
\end{aligned}
$$

Here, the covariant derivatives are defined by

$$
\begin{aligned}
& D_{\mu}=\partial_{\mu}+i g T^{a} V_{a \mu}+i g^{\prime} X T^{9} B_{\mu}, \quad \bar{D}_{\mu}=\partial_{\mu}-i g T^{a *} V_{a \mu}+i g^{\prime} X T^{9} B_{\mu}, \\
& D_{1 \mu}=\partial_{\mu}+i g^{\prime} X T^{9} B_{\mu}, \quad D_{\mu}^{c} \lambda_{c}^{a}=\partial_{\mu} \lambda_{c}^{a}-g_{s} f^{a b c} g_{\mu}^{b} \lambda_{c}^{c}, \\
& D_{\mu}^{L} \lambda_{V}^{a}=\partial_{\mu} \lambda_{V}^{a}-g f^{a b c} V_{\mu}^{b} \lambda_{V}^{c} .
\end{aligned}
$$

The relevant interactions are therefore given by [13]:

$$
\begin{aligned}
\mathcal{L}_{\text {interaction }}= & \mathcal{L}_{l l V}+\mathcal{L}_{\tilde{l} \tilde{l} V}+\mathcal{L}_{l \tilde{l} \tilde{V}}+\mathcal{L}_{\tilde{l} \tilde{l} V V}+\mathcal{L}_{q q V}+\mathcal{L}_{\tilde{q} \tilde{q} V}+\mathcal{L}_{q \tilde{q} \tilde{V}} \\
& +\mathcal{L}_{\tilde{q} \tilde{q} V V}+\mathcal{L}_{H \tilde{H} \tilde{V}}+\mathcal{L}_{l l H}+\mathcal{L}_{l \tilde{l} \tilde{H}}+\mathcal{L}_{l \tilde{H} H}+\mathcal{L}_{\tilde{l} \tilde{H} \tilde{H}} \\
& +\mathcal{L}_{q q H}+\mathcal{L}_{q \tilde{q} \tilde{H}}+\mathcal{L}_{l q \tilde{q}}+\mathcal{L}_{q q \tilde{q}}+\mathcal{L}_{q q \tilde{l}}+V_{\text {scalar }}
\end{aligned}
$$

where

$$
\mathcal{L}_{l l V}=-\frac{g}{2} \bar{L} \bar{\sigma}^{\mu} \lambda^{a} L V_{\mu}^{a}-\frac{g^{\prime}}{\sqrt{6}}\left(-\frac{1}{3}\right) \bar{L} \bar{\sigma}^{\mu} L B_{\mu}-\frac{g^{\prime}}{\sqrt{6}} \bar{l}^{c} \bar{\sigma}^{\mu} l^{c} B_{\mu},
$$




$$
\begin{aligned}
& \mathcal{L}_{\tilde{l} \tilde{l} V}=\frac{i g}{2}\left[\partial^{\mu} \overline{\tilde{L}} \lambda^{a} \tilde{L}-\overline{\tilde{L}} \lambda^{a} \partial^{\mu} \tilde{L}\right] V_{\mu}^{a} \\
& +\frac{i g^{\prime}}{\sqrt{6}}\left[-\frac{1}{3}\left(\partial^{\mu} \overline{\tilde{L}} \tilde{L}-\overline{\tilde{L}} \partial^{\mu} \tilde{L}\right)+\left(\partial^{\mu} \overline{\tilde{l}} \tilde{l}^{c}-\overline{\tilde{l}}^{c} \partial^{\mu} \tilde{l}^{c}\right)\right] B_{\mu}, \\
& \mathcal{L}_{l \tilde{l} \tilde{V}}=-\frac{i g}{\sqrt{2}}\left(\bar{L} \lambda^{a} \tilde{L} \bar{\lambda}_{V}^{a}-\overline{\tilde{L}} \lambda^{a} L \lambda_{V}^{a}\right) \\
& -i g^{\prime} \sqrt{\frac{1}{3}}\left[-\frac{1}{3}\left(\bar{L} \tilde{L} \bar{\lambda}_{B}-\overline{\tilde{L}} L \lambda_{B}\right)+\left(\bar{l} c \tilde{l}^{c} \bar{\lambda}_{B}-\overline{\tilde{l}}^{c} l^{c} \lambda_{B}\right)\right], \\
& \mathcal{L}_{\tilde{l} \tilde{l} V V}=\frac{1}{4}\left[g^{2} V_{\mu}^{a} V^{b \mu} \overline{\tilde{L}} \lambda^{a} \lambda^{b} \tilde{L}+\frac{2}{27} g^{\prime 2} V^{\mu} V_{\mu} \overline{\tilde{L}} \tilde{L}-\frac{2}{3} \sqrt{\frac{2}{3}} g g^{\prime} V_{\mu}^{a} B^{\mu} \overline{\tilde{L}} \lambda^{a} \tilde{L}\right] \\
& +\frac{g^{\prime 2}}{6} B^{\mu} B_{\mu} \overline{\bar{l}} \tilde{l}^{c} \\
& \mathcal{L}_{q q V}=-\frac{g_{s}}{2}\left(\bar{Q}_{i} \bar{\sigma}^{\mu} \lambda^{a} Q_{i}-\bar{u}_{i}^{c} \bar{\sigma}^{\mu} \lambda^{* a} u_{i}^{c}-\bar{d}_{i}^{c} \bar{\sigma}^{\mu} \lambda^{* a} d_{i}^{c}-\bar{u}^{\prime c} \bar{\sigma}^{\mu} \lambda^{* a} u^{\prime c}-\bar{d}_{\beta}^{\prime c} \bar{\sigma}^{\mu} \lambda^{* a} d_{\beta}^{\prime c}\right) g_{\mu}^{a} \\
& -\frac{g}{2}\left(\bar{Q}_{1} \bar{\sigma}^{\mu} \lambda^{a} Q_{1}-\bar{Q}_{\alpha} \bar{\sigma}^{\mu} \lambda^{* a} Q_{\alpha}\right) V_{\mu}^{a} \\
& -\frac{g^{\prime}}{\sqrt{6}}\left[\frac{1}{3} \bar{Q}_{1} \bar{\sigma}^{\mu} Q_{1}-\frac{2}{3} \bar{u}_{i}^{c} \bar{\sigma}^{\mu} u_{i}^{c}+\frac{1}{3} \bar{d}_{i}^{c} \bar{\sigma}^{\mu} d_{i}^{c}-\frac{2}{3} \bar{u}^{\prime c} \bar{\sigma}^{\mu} u^{\prime c}+\frac{1}{3} \bar{d}_{\beta}^{\prime c} \bar{\sigma}^{\mu} d_{\beta}^{\prime c}\right] B_{\mu}, \\
& \mathcal{L}_{\tilde{q} \tilde{q} V}=\frac{i g_{s}}{2}\left(\partial^{\mu} \overline{\tilde{Q}}_{i} \lambda^{a} \tilde{Q}_{i}-\overline{\tilde{Q}}_{i} \lambda^{a} \partial^{\mu} \tilde{Q}_{i}-\partial^{\mu} \overline{\tilde{u}}_{i}^{c} \lambda^{* a} \tilde{u}_{i}^{c}+\overline{\tilde{u}}_{i}^{c} \lambda^{* a} \partial^{\mu} \tilde{u}_{i}^{c}-\partial^{\mu} \overline{\tilde{d}}_{i}^{c} \lambda^{* a} \tilde{d}_{i}^{c}\right. \\
& \left.+\overline{\tilde{d}}_{i}^{c} \lambda^{* a} \partial^{\mu} \tilde{d}_{i}^{c}-\partial^{\mu} \overline{\tilde{u}}^{\prime c} \lambda^{* a} \tilde{u}^{\prime c}+\overline{\tilde{u}}^{\prime c} \lambda^{* a} \partial^{\mu} \tilde{u}^{\prime c}-\partial^{\mu} \overline{\tilde{d}}_{\beta}^{\prime c} \lambda^{* a} \tilde{d}_{\beta}^{\prime c}+\overline{\tilde{d}}_{\beta}^{\prime c} \lambda^{* a} \partial^{\mu} \tilde{d}_{\beta}^{\prime c}\right) g_{\mu}^{a} \\
& +\frac{i g}{2}\left(\partial^{\mu} \overline{\tilde{Q}}_{1} \lambda^{a} \tilde{Q}_{1}-\overline{\tilde{Q}}_{1} \lambda^{a} \partial^{\mu} \tilde{Q}_{1}-\partial^{\mu} \overline{\tilde{Q}}_{\alpha} \lambda^{* a} \tilde{Q}_{\alpha}+\overline{\tilde{Q}}_{\alpha} \lambda^{* a} \partial^{\mu} \tilde{Q}_{\alpha}\right) V_{\mu}^{a} \\
& +\frac{i g^{\prime}}{\sqrt{6}}\left[\frac{1}{3}\left(\partial^{\mu} \overline{\tilde{Q}}_{1} \tilde{Q}_{1}-\overline{\tilde{Q}}_{1} \partial^{\mu} \tilde{Q}_{1}\right)-\frac{2}{3}\left(\partial^{\mu} \overline{\tilde{u}}_{i}^{c} \tilde{u}_{i}^{c}-\overline{\tilde{u}}_{i}^{c} \partial^{\mu} \tilde{u}_{i}^{c}\right)\right. \\
& -\frac{2}{3}\left(\partial^{\mu} \overline{\tilde{u}}^{\prime c} \tilde{u}^{\prime c}-\overline{\tilde{u}}^{\prime c} \partial^{\mu} \tilde{u}^{c}\right)+\frac{1}{3}\left(\partial^{\mu} \overline{\tilde{d}}_{i}^{c} \tilde{d}_{i}^{c}-\overline{\tilde{d}}_{i}^{c} \partial^{\mu} \tilde{d}_{i}^{c}\right) \\
& \left.+\frac{1}{3}\left(\partial^{\mu} \overline{\tilde{d}}_{\beta}^{\prime c} \tilde{d}_{\beta}^{\prime c}-\overline{\tilde{d}}_{\beta}^{\prime c} \partial^{\mu} \tilde{d}_{\beta}^{\prime c}\right)\right] B_{\mu} \\
& \mathcal{L}_{q \tilde{q} \tilde{V}}=-\frac{i g_{s}}{\sqrt{2}}\left[\left(\bar{Q}_{i} \lambda^{a} \tilde{Q}_{i}-\bar{u}_{i}^{c} \lambda^{* a} \tilde{u}_{i}^{c}-\bar{d}_{i}^{c} \lambda^{* a} \tilde{d}_{i}^{c}-\bar{u}^{\prime c} \lambda^{* a} \tilde{u}^{\prime c}-\bar{d}_{\beta}^{\prime c} \lambda^{* a} \tilde{d}_{\beta}^{\prime c}\right) \bar{\lambda}_{c}^{a}\right. \\
& \left.-\left(\overline{\tilde{Q}}_{i} \lambda^{a} Q_{i}-\overline{\tilde{u}}_{i}^{c} \lambda^{* a} u_{i}^{c}-\overline{\tilde{d}}_{i}^{c} \lambda^{* a} d_{i}^{c}-\overline{\tilde{u}}^{\prime c} \lambda^{* a} u^{\prime c}-\overline{\tilde{d}}_{\beta}^{\prime c} \lambda^{* a} d_{\beta}^{\prime c}\right) \lambda_{c}^{a}\right] \\
& -\frac{i g}{\sqrt{2}}\left[\left(\bar{Q}_{1} \lambda^{a} \tilde{Q}_{1}-\bar{Q}_{\alpha} \lambda^{* a} \tilde{Q}_{\alpha}\right) \bar{\lambda}_{V}^{a}-\left(\overline{\tilde{Q}}_{1} \lambda^{a} Q_{1}-\overline{\tilde{Q}}_{\alpha} \lambda^{* a} Q_{\alpha}\right) \lambda_{V}^{a}\right] \\
& -\frac{i g^{\prime}}{\sqrt{3}}\left[\left(\frac{1}{3} \bar{Q}_{1} \tilde{Q}_{1}-\frac{2}{3} \bar{u}_{i}^{c} \tilde{u}_{i}^{c}+\frac{1}{3} \bar{d}_{i}^{c} \tilde{d}_{i}^{c}-\frac{2}{3} \bar{u}^{\prime c} \tilde{u}^{\prime c}+\frac{1}{3} \bar{d}_{\beta}^{\prime c} \tilde{d}_{\beta}^{\prime c}\right) \bar{\lambda}_{B}\right. \\
& \left.-\left(\frac{1}{3} \overline{\tilde{Q}}_{1} Q_{1}-\frac{2}{3} \overline{\tilde{u}}_{i}^{c} u_{i}^{c}+\frac{1}{3} \overline{\tilde{d}}_{i}^{c} d_{i}^{c}-\frac{2}{3} \overline{\tilde{u}}^{\prime c} u^{\prime c}+\frac{1}{3} \overline{\tilde{d}}_{\beta}^{\prime c} d_{\beta}^{\prime c}\right) \lambda_{B}\right] \text {, }
\end{aligned}
$$




$$
\begin{aligned}
& \mathcal{L}_{\tilde{q} \tilde{q} V V}=\frac{1}{4}\left\{g _ { s } ^ { 2 } \left[\overline{\tilde{Q}}_{i} \lambda^{a} \lambda^{b} \tilde{Q}_{i}+\overline{\tilde{u}}_{i}^{c} \lambda^{* a} \lambda^{* b} \tilde{u}_{i}^{c}+\overline{\tilde{d}}_{i}^{c} \lambda^{* a} \lambda^{* b} \tilde{d}_{i}^{c}+\overline{\tilde{u}}^{\prime c} \lambda^{* a} \lambda^{* b} \tilde{u}^{\prime c}\right.\right. \\
& \left.+\overline{\tilde{d}}_{\beta}^{\prime c} \lambda^{* a} \lambda^{* b} \tilde{d}_{\beta}^{\prime c}\right] g_{\mu}^{a} g^{b \mu}+g^{2}\left[\overline{\tilde{Q}}_{\alpha} \lambda^{* a} \lambda^{* b} \tilde{Q}_{\alpha}+\overline{\tilde{Q}}_{1} \lambda^{a} \lambda^{b} \tilde{Q}_{1}\right] V_{\mu}^{a} V^{b \mu} \\
& +\frac{2}{3} g^{\prime 2}\left[\left(\frac{1}{3}\right)^{2} \overline{\tilde{Q}}_{1} \tilde{Q}_{1}+\left(\frac{-2}{3}\right)^{2} \overline{\tilde{u}}_{i}^{c} \tilde{u}_{i}^{c}+\left(\frac{1}{3}\right)^{2} \overline{\tilde{d}}^{c}{ }_{i} \tilde{d}^{c}{ }_{i}\right. \\
& \left.+\left(\frac{-2}{3}\right)^{2} \overline{\tilde{u}}^{\prime c} \tilde{u}^{\prime c}+\left(\frac{1}{3}\right)^{2} \overline{\tilde{d}}_{\alpha}^{\prime c} \tilde{d}_{\alpha}^{\prime c}\right] B_{\mu} B^{\mu} \\
& +2 g_{s} g\left[\overline{\tilde{Q}}_{1} \lambda^{a} \lambda^{b} \tilde{Q}_{1}-\overline{\tilde{Q}}_{\alpha} \lambda^{a} \lambda^{* b} \tilde{Q}_{\alpha}\right] g_{\mu}^{a} V^{b \mu}+\frac{2}{3} \sqrt{\frac{2}{3}} g g^{\prime} \overline{\tilde{Q}}_{1} \lambda^{a} \tilde{Q}_{1} V_{\mu}^{a} B^{\mu} \\
& +2 \sqrt{\frac{2}{3}} g_{s} g^{\prime}\left[\left(\frac{1}{3}\right) \overline{\tilde{Q}}_{1} \lambda^{a} \tilde{Q}_{1}+\left(\frac{-2}{3}\right) \overline{\tilde{u}}_{i}^{c} \lambda^{a} \tilde{u}_{i}^{c}+\left(\frac{1}{3}\right) \overline{\tilde{d}}_{i}^{c} \lambda^{a} \tilde{d}^{c}{ }_{i}\right. \\
& \left.\left.+\left(\frac{-2}{3}\right) \overline{\tilde{u}}^{\prime c} \lambda^{a} \tilde{u}^{\prime c}+\left(\frac{1}{3}\right) \overline{\tilde{d}}_{\alpha}^{\prime c} \lambda^{a} \tilde{d}_{\alpha}^{\prime c}\right] g_{\mu}^{a} B^{\mu}\right\}, \\
& \mathcal{L}_{H \tilde{H} \tilde{V}}=-\frac{i g}{\sqrt{2}}\left[\overline{\tilde{\rho}} \lambda^{a} \rho \bar{\lambda}_{V}^{a}-\bar{\rho} \lambda^{a} \tilde{\rho} \lambda_{V}^{a}+\overline{\tilde{\chi}} \lambda^{a} \chi \bar{\lambda}_{V}^{a}-\bar{\chi} \lambda^{a} \tilde{\chi} \lambda_{V}^{a}-\overline{\tilde{\rho}} \lambda^{* a} \rho^{\prime} \bar{\lambda}_{V}^{a}\right. \\
& \left.+\bar{\rho}^{\prime} \lambda^{* a} \tilde{\rho}^{\prime} \lambda_{V}^{a}-\overline{\tilde{\chi}}^{\prime} \lambda^{* a} \chi^{\prime} \bar{\lambda}_{V}^{a}+\bar{\chi}^{\prime} \lambda^{* a} \tilde{\chi}^{\prime} \lambda_{A}^{a}\right] \\
& -\frac{i g^{\prime}}{\sqrt{3}}\left[-\frac{1}{3}\left(\overline{\tilde{\chi}} \chi \bar{\lambda}_{B}-\bar{\chi} \tilde{\chi} \lambda_{B}\right)+\frac{1}{3}\left(\overline{\tilde{\chi}}^{\prime} \chi^{\prime} \bar{\lambda}_{B}-\bar{\chi}^{\prime} \tilde{\chi}^{\prime} \lambda_{B}\right)\right. \\
& \left.+\frac{2}{3}\left(\overline{\tilde{\rho}} \rho \bar{\lambda}_{B}-\bar{\rho} \tilde{\rho} \lambda_{B}\right)-\frac{2}{3}\left(\overline{\tilde{\rho}} \rho^{\prime} \bar{\lambda}_{B}-\bar{\rho}^{\prime} \tilde{\rho}^{\prime} \lambda_{B}\right)\right], \\
& \mathcal{L}_{l \tilde{l} \tilde{H}}=-\frac{\lambda_{1}}{3}\left(L \tilde{\rho}^{\prime} \tilde{l}^{c}+\tilde{L} \tilde{\rho}^{\prime} l^{c}\right)-\frac{\lambda_{4}}{3}(L \tilde{\rho} \tilde{L}+\tilde{L} \tilde{\rho} L), \\
& \mathcal{L}_{l l H}=-\frac{\lambda_{1}}{3} L l^{c} \rho^{\prime}-\frac{\lambda_{4}}{3} L L \rho, \\
& \mathcal{L}_{l \tilde{H} H}=-\frac{\lambda_{2}}{3}(L \tilde{\chi} \rho+\tilde{\rho} L \chi) ; \quad \mathcal{L}_{\tilde{l} \tilde{H} \tilde{H}}=-\frac{\lambda_{2}}{3} \tilde{\chi} \tilde{\rho} \tilde{L}, \\
& \mathcal{L}_{q q H}=-\frac{1}{3}\left[\kappa_{3} u^{c} Q_{\alpha} \rho+\kappa_{3}^{\prime} u^{\prime c} Q_{\alpha} \rho+\kappa_{4 \alpha i} d_{i}^{c} Q_{\alpha} \chi+\kappa_{4 \alpha \beta}^{\prime} d_{\beta}^{\prime c} Q_{\alpha} \chi+\kappa_{1 i} u_{i}^{c} Q_{1} \chi^{\prime}\right. \\
& \left.+\kappa_{1}^{\prime} u^{\prime c} Q_{1} \chi^{\prime}+\kappa_{2 i} d_{i}^{c} Q_{1} \rho^{\prime}+\kappa_{2 \beta}^{\prime} d_{\beta}^{\prime c} Q_{1} \rho^{\prime}\right] \text {, } \\
& \mathcal{L}_{q \tilde{q} \tilde{H}}=-\frac{1}{3}\left[\kappa_{3 \alpha i}\left(Q_{\alpha} \tilde{\rho} \tilde{u}_{i}^{c}+\tilde{\rho} u_{i}^{c} \tilde{Q}_{\alpha}\right)+\kappa_{3 \alpha}^{\prime}\left(Q_{\alpha} \tilde{\rho} \tilde{u}^{\prime c}+\tilde{\rho} u^{\prime c} \tilde{Q}_{\alpha}\right)\right. \\
& +\kappa_{4 \alpha i}\left(Q_{\alpha} \tilde{\chi} \tilde{d}_{i}^{c}+\tilde{\chi} d_{i}^{c} \tilde{Q}_{\alpha}\right)+\kappa_{4 \alpha \beta}^{\prime}\left(Q_{\alpha} \tilde{\chi} \tilde{d}_{\beta}^{\prime c}+\tilde{\chi} d_{\beta}^{\prime c} \tilde{Q}_{\alpha}\right) \\
& +\kappa_{1 i}\left(Q_{1} \tilde{\chi}^{\prime} \tilde{u}_{i}^{c}+\tilde{\chi}^{\prime} u_{i}^{c} \tilde{Q}_{1}\right)+\kappa_{1}^{\prime}\left(Q_{1} \tilde{\chi}^{\prime} \tilde{u}^{\prime c}+\tilde{\chi}^{\prime} u^{\prime c} \tilde{Q}_{1}\right) \\
& \left.+\kappa_{2 i}\left(Q_{1} \tilde{\rho}^{\prime} \tilde{d}_{i}^{c}+\tilde{\rho}^{\prime} d_{i}^{c} \tilde{Q}_{1}\right)+\kappa_{2 \beta}^{\prime}\left(Q_{1} \tilde{\rho}^{\prime} \tilde{d}_{\beta}^{\prime c}+\tilde{\rho}^{\prime} d_{\beta}^{\prime c} \tilde{Q}_{1}\right)\right] \text {, } \\
& \mathcal{L}_{l q \tilde{q}}=-\frac{\xi_{7}}{3}\left(L Q_{\alpha} \tilde{d}^{c}+d^{c} L \tilde{Q}_{\alpha}\right)-\frac{\xi_{8}}{3}\left(L Q_{\alpha} \tilde{d}^{\prime c}+d^{\prime c} L \tilde{Q}_{\alpha}\right) \text {, } \\
& \mathcal{L}_{\tilde{l} q q}=-\frac{\xi_{7}}{3} Q_{\alpha} d^{c} \tilde{L}-\frac{\xi_{8}}{3} Q_{\alpha} d^{\prime c} \tilde{L},
\end{aligned}
$$




$$
\begin{aligned}
\mathcal{L}_{q q \tilde{q}}= & -\frac{1}{3}\left[f_{1} Q Q \tilde{Q}+\xi_{1}\left(d^{c} d^{\prime c} \tilde{u}^{c}+\tilde{d}^{c} d^{\prime c} u^{c}+d^{c} \tilde{d}^{\prime c} u^{c}\right)\right. \\
& +\xi_{2}\left(d^{c} d^{\prime c} \tilde{u}^{\prime c}+\tilde{d}^{c} d^{\prime c} u^{\prime c}+d^{c} \tilde{d}^{\prime c} u^{\prime c}\right)+\xi_{3}\left(d^{c} d^{c} \tilde{u}^{c}+\tilde{d}^{c} d^{c} u^{c}+d^{c} \tilde{d}^{c} u^{c}\right) \\
& +\xi_{4}\left(d^{c} d^{c} \tilde{u}^{\prime c}+\tilde{d}^{c} d^{c} u^{\prime c}+d^{c} \tilde{d}^{c} u^{\prime c}\right)+\xi_{5}\left(d^{\prime c} d^{\prime c} \tilde{u}^{c}+\tilde{d}^{\prime c} d^{\prime c} u^{c}+d^{\prime c} \tilde{d}^{\prime c} u^{c}\right) \\
& \left.+\xi_{6}\left(d^{\prime c} d^{\prime c} \tilde{u}^{\prime c}+\tilde{d}^{\prime c} d^{\prime c} u^{\prime c}+d^{\prime c} \tilde{d}^{\prime c} u^{\prime c}\right)\right] .
\end{aligned}
$$

The scalar potential $V_{\text {scalar }}$ has a form

$$
V_{\text {scalar }}=V_{F}+V_{D}
$$

To find $V_{F}$ and $V_{D}$, using firstly the Euler-Lagrangian equations for the auxiliary fields, we obtain

$$
F_{\rho}=-\frac{\mu_{\rho}}{2} \rho^{\prime \dagger}, \quad F_{\chi}=-\frac{\mu_{\chi}}{2} \chi^{\prime \dagger}, \quad F_{\rho^{\prime}}=-\frac{\mu_{\rho}}{2} \rho^{\dagger}, \quad F_{\chi^{\prime}}=-\frac{\mu_{\chi}}{2} \chi^{\dagger},
$$

and

$$
\begin{aligned}
& D^{a}=-\frac{g}{2}\left[\rho^{\dagger} \lambda^{a} \rho+\chi^{\dagger} \lambda^{a} \chi-\rho^{\prime \dagger} \lambda^{* a} \rho^{\prime}-\chi^{\prime \dagger} \lambda^{* a} \chi^{\prime}\right], \\
& D=-\frac{g^{\prime}}{\sqrt{6}}\left[-\frac{1}{3} \chi^{\dagger} \chi+\frac{1}{3} \chi^{\prime \dagger} \chi^{\prime}+\frac{2}{3} \rho^{\dagger} \rho-\frac{2}{3} \rho^{\prime \dagger} \rho^{\prime}\right] .
\end{aligned}
$$

The scalar potential is therefore given by

$$
V_{\text {scalar }}=\frac{1}{2}\left(D^{a} D^{a}+D D\right)+\left|F_{\chi}\right|^{2}+\left|F_{\rho}\right|^{2}+\left|F_{\chi}^{\prime}\right|^{2}+\left|F_{\rho}^{\prime}\right|^{2} .
$$

\subsection{The soft term}

With the help of [20], the most general soft supersymmetry-breaking terms, which do not induce quadratic divergences can be obtained. Such terms, in general, can be categorized as follows:

(i) A scalar field $A$ with the mass term

$$
-m^{2} A^{\dagger} A
$$

(ii) A gaugino $\lambda$ with the mass terms

$$
-\frac{1}{2}\left(M_{\lambda} \lambda^{a} \lambda^{a}+\text { H.c. }\right)
$$

(iii) Finally, trilinear scalar couplings have the forms

$$
\epsilon^{i j k} A_{i} A_{j} A_{k}+\text { H.c. }
$$

In this model, the soft terms are given by

$$
\mathcal{L}_{\text {soft }}=\mathcal{L}_{\mathrm{GMT}}+\mathcal{L}_{\text {scalar }}^{\text {soft }}+\mathcal{L}_{\mathrm{SMT}},
$$

where

$$
\mathcal{L}_{\text {scalar }}^{\text {soft }}=-m_{\rho}^{2} \rho^{\dagger} \rho-m_{\chi}^{2} \chi^{\dagger} \chi-m_{\rho^{\prime}}^{2} \rho^{\prime \dagger} \rho^{\prime}-m_{\chi^{\prime}}^{2} \chi^{\prime \dagger} \chi^{\prime},
$$


and

$$
\begin{aligned}
-\mathcal{L}_{\mathrm{SMT}}= & m_{a L}^{2} \tilde{L}_{a L}^{\dagger} \tilde{L}_{a L}+m_{l a}^{2} \tilde{l}_{a L}^{c \dagger} \tilde{l}_{a L}^{c}+m_{Q 1 L}^{2} \tilde{Q}_{1 L}^{\dagger} \tilde{Q}_{1 L}+m_{Q \alpha L}^{2} \tilde{Q}_{\alpha L}^{\dagger} \tilde{Q}_{\alpha L} \\
& +m_{u_{i}}^{2} \tilde{u}_{i L}^{c \dagger} \tilde{u}_{i L}^{c}+m_{d_{i}}^{2} \tilde{d}_{i L}^{c \dagger} \tilde{d}_{i L}^{c}+m_{u^{\prime}}^{2} \tilde{u}_{L}^{\prime c} \tilde{u}_{L}^{c}+m_{d^{\prime}}^{2} \tilde{d}_{L}^{\prime \dagger} \tilde{d}_{L}^{\prime c}+M_{a}^{\prime 2} \chi^{\dagger} \tilde{L}_{a L} \\
& +\varepsilon_{1 a b} \tilde{L}_{a L} \rho^{\prime} \tilde{l}_{L b}^{c}+\varepsilon_{2 a} \epsilon \tilde{L}_{a L} \chi \varrho+\varepsilon_{3 a b} \epsilon \tilde{L}_{a L} \tilde{L}_{b L} \rho+\varrho_{1 i} \tilde{Q}_{1 L} \chi^{\prime} \tilde{u}_{i L}^{c} \\
& +\varrho_{1}^{\prime} \tilde{Q}_{1 L} \chi^{\prime} \tilde{u}_{i L}^{\prime c}+\varrho_{2 \alpha i} \tilde{Q}_{\alpha L} \rho^{\prime} \tilde{u}_{i L}^{c}+\varrho_{2 \alpha} \tilde{Q}_{\alpha L} \rho^{\prime} \tilde{u}_{L}^{\prime c}+\varrho_{3 i} \tilde{Q}_{1 L} \rho^{\prime} \tilde{d}_{i L}^{c} \\
& +\varrho_{3 i}^{\prime} \tilde{Q}_{1 L} \rho^{\prime} \tilde{d}_{L}^{\prime c}+\varrho_{4 \alpha i} \tilde{Q}_{\alpha L} \chi \tilde{d}_{i L}^{c}+\varrho_{4 \alpha \beta}^{\prime} \tilde{Q}_{\alpha L} \chi \tilde{d}_{\beta L}^{\prime c} \\
& +\varrho_{5 \alpha \beta \gamma} \tilde{Q}_{\alpha L} \tilde{Q}_{\beta L} \tilde{Q}_{\gamma L}+\kappa_{1 i \beta j} \tilde{d}_{i L}^{c} \tilde{d}_{\beta L}^{\prime c} \tilde{u}_{j L}^{c}+\kappa_{2 i \beta} \tilde{d}_{i L}^{c} \tilde{d}_{\beta L}^{\prime c} \tilde{u}_{L}^{\prime c} \\
& +\kappa_{3 i j k} \tilde{d}_{i L}^{c} \tilde{d}_{j L}^{c} \tilde{u}_{k L}^{c}+\kappa_{4 i k} \tilde{d}_{i L}^{c} \tilde{d}_{j L}^{c} \tilde{u}_{L}^{\prime c}+\kappa_{5 \alpha \beta i} \tilde{d}_{\alpha L}^{\prime c} \tilde{d}_{\beta L}^{\prime c} \tilde{u}_{i L}^{c} \\
& +\kappa_{6 \alpha \beta} \tilde{d}_{\alpha L}^{\prime c} \tilde{d}_{\beta L}^{\prime c} \tilde{u}_{L}^{\prime c}+\kappa_{7 a \alpha j} \tilde{L}_{a L} \tilde{Q}_{\alpha L} \tilde{d}_{j L}^{c}+\kappa_{8 a \alpha \beta} \tilde{L}_{a L} \tilde{Q}_{\alpha L} \tilde{d}_{\beta L}^{\prime c} \\
& + \text { H.c. }
\end{aligned}
$$

in order to give appropriate masses to the sfermions.

Finally, the Lagrangian $\mathcal{L}_{\mathrm{GMT}}$ has the same form given in [7]

$$
\mathcal{L}_{\mathrm{GMT}}=-\frac{1}{2}\left[m_{\lambda_{c}} \sum_{b=1}^{8}\left(\lambda_{c}^{b} \lambda_{c}^{b}\right)+m_{\lambda} \sum_{b=1}^{8}\left(\lambda_{V}^{b} \lambda_{V}^{b}\right)+m^{\prime} \lambda_{B} \lambda_{B}+\text { H.c. }\right] .
$$

This part gives masses for the superpartners of gauge bosons.

\section{Gauge bosons}

In Section 6, we will prove that in order to eliminate linear terms in the Higgs potential, one obtains a matching condition $u / w=u^{\prime} / w^{\prime}$. In the following the notation,

$$
t_{\theta} \equiv \frac{u}{w}=\frac{u^{\prime}}{w^{\prime}}
$$

is therefore used, where $s_{\theta} \equiv \sin \theta, t_{\theta} \equiv \tan \theta$, and so forth.

The mass Lagrangian for the gauge bosons can be obtained by

$$
\begin{aligned}
2 \mathcal{L}_{\text {mass }}^{\text {gauge }}= & (u, 0, w)\left(\frac{g}{2} \lambda^{a} V_{a}^{\mu}-\frac{1}{3} \frac{g^{\prime}}{2} \sqrt{\frac{2}{3}} B^{\mu}\right)^{2}(u, 0, w)^{T} \\
& +(0, v, 0)\left(\frac{g}{2} \lambda^{a} V_{a}^{\mu}+\frac{2}{3} \frac{g^{\prime}}{2} \sqrt{\frac{2}{3}} B^{\mu}\right)^{2}(0, v, 0)^{T} \\
& +\left(u^{\prime}, 0, w^{\prime}\right)\left(-\frac{g}{2} \lambda^{a *} V_{a}^{\mu}+\frac{1}{3} \frac{g^{\prime}}{2} \sqrt{\frac{2}{3}} B^{\mu}\right)^{2}\left(u^{\prime}, 0, w^{\prime}\right)^{T} \\
& +\left(0, v^{\prime}, 0\right)\left(-\frac{g}{2} \lambda^{a *} V_{a}^{\mu}-\frac{2}{3} \frac{g^{\prime}}{2} \sqrt{\frac{2}{3}} B^{\mu}\right)^{2}\left(0, v^{\prime}, 0\right)^{T} .
\end{aligned}
$$

Let us define the charged gauge bosons as follows

$$
W_{\mu}^{\prime \pm} \equiv \frac{1}{\sqrt{2}}\left(V_{1 \mu} \mp i V_{2 \mu}\right), \quad Y_{\mu}^{\prime \pm} \equiv \frac{1}{\sqrt{2}}\left(V_{6 \mu} \pm V_{7 \mu}\right)
$$


The mass matrix of the $W_{\mu}^{\prime}$ and $Y_{\mu}^{\prime}$ is obtained then

$$
M_{\text {charged }}^{2}=\frac{g^{2}}{4}\left(\begin{array}{cc}
V^{2}+U^{2} & K \\
K & W^{2}+V^{2}
\end{array}\right),
$$

where

$$
\begin{aligned}
& V^{2} \equiv v^{2}+v^{\prime 2}, \quad W^{2} \equiv w^{2}+w^{\prime 2}, \quad U^{2} \equiv u^{2}+u^{\prime 2}=t_{\theta}^{2} W^{2}, \\
& K \equiv u w+u^{\prime} w^{\prime}=t_{\theta} W^{2}, \quad t \equiv g^{\prime} / g .
\end{aligned}
$$

This matrix gives the eigenstates which are, respectively, the SM-like $W^{ \pm}$and new gauge boson $Y^{ \pm}$:

$$
W_{\mu}=c_{\theta} W_{\mu}^{\prime}-s_{\theta} Y_{\mu}^{\prime}, \quad Y_{\mu}=s_{\theta} W_{\mu}^{\prime}+c_{\theta} Y_{\mu}^{\prime},
$$

with the respective eigenvalues:

$$
m_{W}^{2}=\frac{g^{2}}{4} V^{2}, \quad m_{Y}^{2}=\frac{g^{2}}{4}\left(V^{2}+U^{2}+W^{2}\right) .
$$

Therefore, the $\theta$ is the mixing angle of $W^{\prime}-Y^{\prime}$, which is the same as in the case of nonsupersymmetric model [9]. Because of the constraint (15), the mass of $W$ boson is identified with those of the SM, that is

$$
\sqrt{v^{2}+v^{\prime 2}} \equiv v_{\text {weak }}=246 \mathrm{GeV} \text {. }
$$

For the remaining gauge vectors $\left(V_{3}, V_{8}, B, V_{4}, V_{5}\right)$, the mass matrix in this basis is given by

$$
M_{\text {neutral }}^{2}=\left(\begin{array}{cc}
M_{\text {mixing }}^{2} & 0 \\
0 & M_{V_{5}}^{2}
\end{array}\right),
$$

where $V_{5}$ is decoupled with the mass

$$
M_{V_{5}}^{2} \equiv \frac{g^{2}}{4}\left(W^{2}+U^{2}\right),
$$

while the mixing part $M_{\text {mixing }}^{2}$ of $\left(V_{3}, V_{8}, B, V_{4}\right)$ is equal to

$$
\frac{g^{2}}{4}\left(\begin{array}{cccc}
U^{2}+V^{2} & \frac{1}{\sqrt{3}}\left(U^{2}-V^{2}\right) & -\frac{2 t}{3 \sqrt{6}}\left(U^{2}+2 V^{2}\right) & K \\
& \frac{1}{3}\left(V^{2}+U^{2}+4 W^{2}\right) & \frac{\sqrt{2} t}{9}\left(2 V^{2}+2 W^{2}-U^{2}\right) & -\frac{1}{\sqrt{3}} K \\
& & \frac{2 t^{2}}{27}\left(4 V^{2}+U^{2}+W^{2}\right) & -\frac{4 t}{3 \sqrt{6}} K \\
& & & U^{2}+W^{2}
\end{array}\right) .
$$

As in the non-supersymmetric version, it can be checked that the matrix (52) contains two exact eigenvalues, the photon $A_{\mu}$ and new $V_{4 \mu}^{\prime} \sim V_{4 \mu}$, such as

$$
M_{\gamma}^{2}=0, \quad M_{V_{4}^{\prime}}^{2}=\frac{g^{2}}{4}\left(U^{2}+W^{2}\right) .
$$

Due to the fact that $V_{4}^{\prime}$ and $V_{5}$ gain the same mass [cf. (53) and (51)], it is worth noting that these boson vectors have to be combined to produce the following physical state [9]

$$
X_{\mu}^{0} \equiv \frac{1}{\sqrt{2}}\left(V_{4 \mu}^{\prime}-i V_{5 \mu}\right)
$$


with the mass

$$
m_{X}^{2}=\frac{g^{2}}{4}\left(U^{2}+W^{2}\right)
$$

To look for eigenstates corresponding to eigenvalues in (53), we will separate the square mass matrix (52) into two parts such as

$$
M_{\text {mixing }}^{2}=M_{1}^{2}+M_{2}^{2},
$$

with

$$
M_{1}^{2}=\frac{g^{2}}{4}\left(\begin{array}{cccc}
u^{2}+v^{2} & \frac{1}{\sqrt{3}}\left(u^{2}-v^{2}\right) & -\frac{2 t}{3 \sqrt{6}}\left(u^{2}+2 v^{2}\right) & u w \\
& \frac{1}{3}\left(v^{2}+u^{2}+4 w^{2}\right) & \frac{\sqrt{2} t}{9}\left(2 v^{2}+2 w^{2}-u^{2}\right) & -\frac{1}{\sqrt{3}} u w \\
& \frac{2 t^{2}}{27}\left(4 v^{2}+u^{2}+w^{2}\right) & -\frac{4 t}{3 \sqrt{6}} u w \\
& & u^{2}+w^{2}
\end{array}\right)
$$

and $M_{2}^{2}$ equal to

$$
\frac{g^{2}}{4}\left(\begin{array}{cccc}
u^{\prime 2}+v^{\prime 2} & \frac{1}{\sqrt{3}}\left(u^{\prime 2}-v^{\prime 2}\right) & -\frac{2 t}{3 \sqrt{6}}\left(u^{\prime 2}+2 v^{2 \prime}\right) & u^{\prime} w^{\prime} \\
& \frac{1}{3}\left(v^{2 \prime}+u^{2 \prime}+4 w^{2 \prime}\right) & \frac{\sqrt{2} t}{9}\left(2 v^{\prime 2}+2 w^{2 \prime}-u^{2 \prime}\right) & -\frac{1}{\sqrt{3}} u^{\prime} w^{\prime} \\
& \frac{2 t^{2}}{27}\left(4 v^{2 \prime}+u^{2 \prime}+w^{2 \prime}\right) & -\frac{4 t}{3 \sqrt{6}} u^{\prime} w^{\prime} \\
& & u^{2 \prime}+w^{2 \prime}
\end{array}\right) .
$$

It is easy to realize that $M_{1}^{2}$ and $M_{2}^{2}$ have the same form as in non-supersymmetric version in Ref. [9]. Therefore $M_{1}^{2}$ and $M_{2}^{2}$, respectively, contain the two eigenvalues as follows

$$
\lambda_{1}=0, \quad \lambda_{2}=\frac{g^{2}}{4}\left(u^{2}+w^{2}\right), \quad \lambda_{1}^{\prime}=0, \quad \lambda_{2}^{\prime}=\frac{g^{2}}{4}\left(u^{\prime 2}+w^{\prime 2}\right) .
$$

This means also that the matrix $M_{\text {mixing }}^{2}$ contains a massless eigenstate corresponding to the photon [21]

$$
A_{\mu}^{T}=\frac{1}{\sqrt{18+4 t^{2}}}(\sqrt{3} t,-t, 3 \sqrt{2}, 0) .
$$

Before seeking the eigenstate of $M_{V_{4}^{\prime}}^{2}$, we note that the eigenstates according to eigenvalues $\lambda_{2}$ and $\lambda_{2}^{\prime}$ are, respectively, given by

$$
\begin{aligned}
& V_{4 \mu \lambda_{2}}^{\prime T}=\frac{1}{\sqrt{1+4 \Lambda^{2}}}(\Lambda, \sqrt{3} \Lambda, 0,1), \quad \Lambda \equiv \frac{2 u w}{w^{2}-u^{2}} \\
& V_{4 \mu \lambda_{2}^{\prime}}^{\prime T}=\frac{1}{\sqrt{1+4 \Lambda^{\prime 2}}}\left(\Lambda^{\prime}, \sqrt{3} \Lambda^{\prime}, 0,1\right), \quad \Lambda^{\prime} \equiv \frac{2 u^{\prime} w^{\prime}}{w^{\prime 2}-u^{\prime 2}} .
\end{aligned}
$$

Because of the condition (42), we get a beautiful result

$$
V_{4 \mu}^{\prime T}=V_{4 \mu \lambda_{2}}^{\prime T}=V_{4 \mu \lambda_{2}^{\prime}}^{\prime T}=\frac{1}{\sqrt{1+4 t_{2 \theta}^{2}}}\left(t_{2 \theta}, \sqrt{3} t_{2 \theta}, 0,1\right) .
$$

These results are the same as in Ref. [9] of the non-supersymmetric version. 
The eigenvectors $A_{\mu}$ and $V_{4 \mu}^{\prime}$ can be rewritten as follows

$$
\begin{aligned}
& A_{\mu}=s_{W} V_{3 \mu}+c_{W}\left(-\frac{t_{W}}{\sqrt{3}} V_{8 \mu}+\sqrt{1-\frac{t_{W}^{2}}{3}}\right) B_{\mu}, \\
& V_{4 \mu}^{\prime}=\frac{t_{2 \theta}}{\sqrt{1+4 t_{2 \theta}^{2}}} V_{3 \mu}+\frac{\sqrt{3} t_{2 \theta}}{\sqrt{1+4 t_{2 \theta}^{2}}} V_{8 \mu}+\frac{1}{\sqrt{1+4 t_{2 \theta}^{2}}} V_{4 \mu},
\end{aligned}
$$

where $s_{W} \equiv \sin \theta_{W}=\sqrt{3 t^{2} /\left(18+4 t^{2}\right)}$ [21]. Further, let us define two gauge vectors

$$
\begin{aligned}
& Z_{\mu}=c_{W} V_{3 \mu}-s_{W}\left(-\frac{t_{W}}{\sqrt{3}} V_{8 \mu}+\sqrt{1-\frac{t_{W}^{2}}{3}}\right) B_{\mu}, \\
& Z_{\mu}^{\prime}=\frac{t_{W}}{\sqrt{3}} B_{\mu}+\sqrt{1-\frac{t_{W}^{2}}{3}} V_{8 \mu},
\end{aligned}
$$

which are orthogonal to $A_{\mu}$. To look for two last eigenstates, we will use the argument in [9] to define

$$
\begin{aligned}
& Z_{1 \mu}=c_{\theta^{\prime}} Z_{\mu}-s_{\theta^{\prime}}\left[t_{\theta^{\prime}} \sqrt{4 c_{W}^{2}-1} Z_{\mu}^{\prime}+\sqrt{1-t_{\theta^{\prime}}^{2}\left(4 c_{W}^{2}-1\right)} V_{4 \mu}\right], \\
& Z_{1 \mu}^{\prime}=\sqrt{1-t_{\theta^{\prime}}^{2}\left(4 c_{W}^{2}-1\right)} Z_{\mu}^{\prime}-t_{\theta^{\prime}} \sqrt{4 c_{W}^{2}-1} V_{4 \mu},
\end{aligned}
$$

where $s_{\theta^{\prime}} \equiv t_{2 \theta} / c_{W} / \sqrt{1+4 t_{2 \theta}^{2}}$. Then, in the base of $\left(A_{\mu}, Z_{1 \mu}, Z_{1 \mu}^{\prime}, V_{4 \mu}^{\prime}\right)$, the squared-mass matrix $M_{\text {neutral }}^{2}$ becomes

$$
M_{\text {neutral }}^{\prime 2}=\left(\begin{array}{cccc}
0 & 0 & 0 & 0 \\
0 & m_{Z_{1}}^{2} & m_{Z_{1} Z_{1}^{\prime}}^{2} & 0 \\
0 & m_{Z_{1} Z_{1}^{\prime}}^{2} & m_{Z_{1}^{\prime}}^{2} & 0 \\
0 & 0 & 0 & \frac{g^{2}}{4}\left(W^{2}+U^{2}\right)
\end{array}\right)
$$

with

$$
\begin{aligned}
m_{Z_{1}}^{2}= & g^{2} \\
m_{Z_{1} Z_{1}^{\prime}}^{2}= & g^{2}\left\{\sqrt{1+4 t_{2 \theta}^{2}}\left[c_{2 W}+\left(3-4 s_{W}^{2}\right) t_{2 \theta}^{2}\right] U^{2}-V^{2}-\left(3-4 s_{W}^{2}\right) t_{2 \theta}^{2} W^{2}\right\} \\
& \times\left\{4 \sqrt{3-4 s_{W}^{2}}\left[c_{W}^{2}+\left(3-4 s_{W}^{2}\right) t_{2 \theta}^{2}\right]\right\}^{-1}, \\
m_{Z_{1}^{\prime}}^{2}= & g^{2}\left\{\left[c_{2 W}^{2}+\left(3-4 s_{2 W}^{2}\right) t_{2 \theta}^{2}\right] U^{2}+V^{2}\right. \\
+ & {\left.\left[4 c_{W}^{2}+\left(1+4 c_{W}^{2}\right)\left(3-4 s_{W}^{2}\right) t_{2 \theta}^{2}\right] W^{2}\right\}\left\{4\left(3-4 s_{W}^{2}\right)\left[c_{W}^{2}+\left(3-4 s_{W}^{2}\right) t_{2 \theta}^{2}\right]\right\}^{-1} . }
\end{aligned}
$$

The elements of this matrix have the same form as of non-supersymmetric version [9], where the results are obtained with replacement of $u, v, w$ by $U, V, W$. The last two eigenstates and masses of the neutral gauge bosons are given as in [9]. 
To finish this section, we mention again that the matrix of neutral gauge boson mixing is separated into two terms and one of them is the same as in the non-supersymmetric version. Because of the relation among the VEVs $\omega, \omega^{\prime}$ and $u, u^{\prime}$, the exact diagonalization was easily performed. Here, the gauge boson identification is the same as in non-supersymmetric case. This means that the imaginary part of the non-Hermitian bilepton $X^{0}$ is decoupled, while its real part has the mixing among the neutral Hermitian gauge bosons such as, the photon, the neutral $Z$ and the extra $Z^{\prime}$.

\section{Lepton and quark sectors}

As in Ref. [15], the R-charge is chosen as follows

$$
\begin{aligned}
& n_{L}=n_{l}=n_{\rho}=n_{\rho^{\prime}}=0, \quad n_{Q_{1}}=n_{u}=n_{u^{\prime}}=\frac{1}{2}, \quad n_{Q_{\alpha}}=n_{d}=n_{d^{\prime}}=-\frac{1}{2}, \\
& n_{\chi}=1, \quad n_{\chi^{\prime}}=-1 .
\end{aligned}
$$

The terms of the superpotential with respect to this R-parity are given by

$$
\begin{aligned}
W= & \frac{\mu_{\chi}}{2} \hat{\chi} \hat{\chi}^{\prime}+\frac{\mu_{\rho}}{2} \hat{\rho} \hat{\rho}^{\prime}+\frac{1}{3}\left[\lambda_{1 a b} \hat{L}_{a L} \hat{\rho}^{\prime} \hat{l}_{b L}^{c}+\lambda_{3 a b} \epsilon \hat{L}_{a L} \hat{L}_{b L} \hat{\rho}\right. \\
& +\kappa_{1 i} \hat{Q}_{1 L} \hat{\chi}^{\prime} \hat{u}_{i L}^{c}+\kappa_{1}^{\prime} \hat{Q}_{1 L} \hat{\chi}^{\prime} \hat{u}_{L}^{\prime c}+\kappa_{2 i} \hat{Q}_{1 L} \hat{\rho}^{\prime} \hat{d}_{i L}^{c}+\kappa_{2 \alpha}^{\prime} \hat{Q}_{1 L} \hat{\rho}^{\prime} \hat{d}_{\alpha L}^{\prime c} \\
& \left.+\kappa_{3 \alpha i} \hat{Q}_{\alpha L} \hat{\rho} \hat{u}_{i L}^{c}+\kappa_{3 \alpha}^{\prime} \hat{Q}_{\alpha L} \hat{\rho} \hat{u}_{L}^{\prime c}+\kappa_{4 \alpha i} \hat{Q}_{\alpha L} \hat{\chi} \hat{d}_{i L}^{c}+\kappa_{4 \alpha \beta}^{\prime} \hat{Q}_{\alpha L} \hat{\chi} \hat{d}_{\beta L}^{\prime c}\right]
\end{aligned}
$$

\subsection{Charged lepton masses}

From the superpotential given in Eq. (68), it is easy to see that the charged leptons gain mass only from the term

$$
-\frac{\lambda_{1 a b}}{3} L_{a L} \rho^{\prime} l_{b L}^{c}+\text { H.c. }
$$

We therefore get mass terms

$$
-\frac{\lambda_{1 a b}}{3}\left(l_{a L} l_{b L}^{c}+\bar{l}_{a L} \bar{l}_{b L}^{c}\right) \frac{v^{\prime}}{\sqrt{2}} .
$$

This mass term can now be rewritten in terms of a $3 \times 3$ matrix $X_{l}$ as follows. Defining the following two column vectors

$$
\left(\psi_{l}^{+}\right)^{T}=\left(l_{1 L}^{c} l_{2 L}^{c} l_{3 L}^{c}\right), \quad\left(\psi_{l}^{-}\right)^{T}=\left(l_{1 L} l_{2 L} l_{3 L}\right),
$$

we can rewrite our mass term as

$$
-\mathcal{L}=\left(\psi_{l}^{-}\right)^{T} X_{l} \psi_{l}^{+}+\text {H.c. }
$$

with

$$
X_{l}=\frac{v^{\prime}}{\sqrt{2}}\left(\begin{array}{ccc}
\frac{\lambda_{111}}{3} & \frac{\lambda_{112}}{3} & \frac{\lambda_{113}}{3} \\
\frac{\lambda_{121}}{3} & \frac{\lambda_{122}}{3} & \frac{\lambda_{123}}{3} \\
\frac{\lambda_{131}}{3} & \frac{\lambda_{132}}{3} & \frac{\lambda_{133}}{3}
\end{array}\right) .
$$

Notice that only VEV of $\rho^{\prime}$ is enough to give the charged leptons masses. 
In order to get the mass eigenstates we perform the following rotation

$$
l_{i}^{+}=V_{i j}^{l}\left(\psi_{l}^{+}\right)_{j}, \quad l_{i}^{-}=U_{i j}^{l}\left(\psi_{l}^{-}\right)_{j}, \quad i, j=1,2,3,
$$

where $V^{l}, U^{l}$ are unitary matrices such that

$$
\underbrace{\psi_{l}^{-T}\left(U^{l}\right)^{T}}_{l_{i}^{-T}} \underbrace{\left(U^{l}\right)^{*} X_{l}\left(V^{l}\right)^{\dagger}}_{\left(\mathcal{M}_{l}\right)_{i j}} \underbrace{V^{l} \psi_{l}^{+}}_{l_{j}^{+}} .
$$

Here $\mathcal{M}_{l}$ is a diagonal matrix with real non-negative entries

$$
\left(\mathcal{M}_{l}\right)_{i j}=\left[\left(U^{l}\right)^{*} X_{l}\left(V^{l}\right)^{\dagger}\right]_{i j}=m_{l_{i}} \delta_{i j}
$$

The charged leptons $l_{i}$ are defined such that their absolute masses increase with increasing $i$.

We have verified that all the charged leptons get mass which are the same as in the usual cases [22].

\subsection{Neutral lepton masses}

Neutrinos get masses from the term

$$
-\frac{\lambda_{3 a b}}{3} L_{a L} L_{b L} \rho+\text { H.c., }
$$

which gives us

$$
-\frac{\lambda_{3 a b}}{3}\left(v_{a L}^{c} v_{b L}-v_{a L} v_{b L}^{c}+\bar{v}_{a L}^{c} \bar{v}_{b L}-\bar{v}_{a L} \bar{v}_{b L}^{c}\right) \rho^{0} .
$$

This mass term can now be rewritten in terms of a $6 \times 6$ matrix $X_{v}$ by defining the following column vector

$$
\left(\psi_{v}^{0}\right)^{T}=\left(\begin{array}{llllll}
v_{1 L} & v_{2 L} & v_{3 L} & v_{1 L}^{c} & v_{2 L}^{c} & v_{3 L}^{c}
\end{array}\right) .
$$

Now we can rewrite our mass term as

$$
-\mathcal{L}=\frac{1}{2}\left[\left(\psi_{\nu}^{0}\right)^{T} X_{\nu} \psi_{\nu}^{0}+\text { H.c. }\right]
$$

with

$$
X_{v}=\frac{v}{\sqrt{2}}\left(\begin{array}{cccccc}
0 & 0 & 0 & 0 & G_{21} & G_{31} \\
0 & 0 & 0 & G_{12} & 0 & G_{32} \\
0 & 0 & 0 & G_{13} & G_{23} & 0 \\
0 & G_{12} & G_{13} & 0 & 0 & 0 \\
G_{21} & 0 & G_{23} & 0 & 0 & 0 \\
G_{31} & G_{32} & 0 & 0 & 0 & 0
\end{array}\right)
$$

where

$$
G_{a b}=\frac{1}{3}\left(\lambda_{3 a b}-\lambda_{3 b a}\right) .
$$

Due to the fact that the above matrix is symmetric, we need only one rotation

$$
v_{i}=V_{i j}^{v}\left(\psi_{v}^{0}\right)_{j}, \quad i, j=1,2, \ldots, 6,
$$


where $V^{v}$ is an unitary matrix such that

$$
\frac{1}{2} \underbrace{\psi_{v}^{0 T}\left(V^{v}\right)^{T}}_{v_{i}^{T}} \underbrace{\left(V^{v}\right)^{*} X_{v}\left(V^{v}\right)^{\dagger}}_{\left(\mathcal{M}_{v}\right)_{i j}} \underbrace{V^{v} \psi_{v}^{0}}_{v_{j}} .
$$

Here $\mathcal{M}_{\nu}$ is a diagonal matrix with real non-negative entries

$$
\left(\mathcal{M}_{v}\right)_{i j}=\left[\left(V^{v}\right)^{*} X_{v}\left(V^{v}\right)^{\dagger}\right]_{i j}=m_{v_{i}} \delta_{i j} .
$$

As before, the neutral leptons $v_{i}$ are defined such that their absolute masses increase with increasing $i$. Due to the fact that $G_{a b}=-G_{b a}$, the mass pattern of this sector is $0,0, m_{v}, m_{v}$, $m_{v}, m_{v}$, where $\sqrt{2} m_{v}=v \sqrt{G_{31}^{2}+G_{32}^{2}+G_{21}^{2}}$. Noting that this mass spectrum is the same as of the non-supersymmetric version. The quantum corrections at one loop level can be constructed as in [12] (see also [23]). This provides a realistic mass spectrum for the neutrinos.

\subsection{Masses of up quarks and down quarks}

The Yukawa couplings responsible for the masses of the up quarks can be obtained by

$$
-\frac{1}{3}\left[\kappa_{1 i} Q_{1 L} u_{i L}^{c} \chi^{\prime}+\kappa_{1}^{\prime} Q_{1 L} u_{L}^{\prime c} \chi^{\prime}+\kappa_{3 \alpha i} Q_{\alpha L} u_{i L}^{c} \rho+\kappa_{3 \alpha}^{\prime} Q_{\alpha L} u_{L}^{\prime c} \rho\right]+\text { H.c. }
$$

This mass term can now be rewritten in terms of a $4 \times 4$ matrix $X_{u}$ as follows. Defining following two column vectors

$$
\left(\psi_{u}^{+}\right)^{T}=\left(u_{1 L}, u_{2 L}, u_{3 L}, u_{L}^{\prime}\right), \quad\left(\psi_{u}^{-}\right)^{T}=\left(u_{1 L}^{c}, u_{2 L}^{c}, u_{3 L}^{c}, u_{L}^{\prime c}\right),
$$

we can rewrite our mass term as

$$
-\mathcal{L}=\left(\psi_{u}^{-}\right)^{T} X_{u} \psi_{u}^{+}+\text {H.c. },
$$

with

$$
X_{u}=\frac{1}{3 \sqrt{2}}\left(\begin{array}{cccc}
\kappa_{11} u^{\prime} & \kappa_{12} u^{\prime} & \kappa_{13} u^{\prime} & \kappa_{1}^{\prime} u^{\prime} \\
-\kappa_{321} v & -\kappa_{322} v & -\kappa_{323} v & -\kappa_{32}^{\prime} v \\
-\kappa_{331} v & -\kappa_{332} v & -\kappa_{333} v & -\kappa_{33}^{\prime} v \\
\kappa_{11} w^{\prime} & \kappa_{12} w^{\prime} & \kappa_{13} w^{\prime} & \kappa_{1}^{\prime} w^{\prime}
\end{array}\right) .
$$

It is easily to see that the first row and the last row in the mass matrix (89) are proportional. This means that we obtain one massless particle in the mass spectrum of the up quarks. This problem is the same as in the non-supersymmetric version [11].

Similarly, the Yukawa couplings for the down-quark masses are given by

$$
-\frac{1}{3}\left[\kappa_{2 i} Q_{1 L} d_{i L}^{c} \rho^{\prime}+\kappa_{2 \beta}^{\prime} Q_{1 L} d_{\beta L}^{\prime c} \rho^{\prime}+\kappa_{4 \alpha i} Q_{\alpha L} d_{i L}^{c} \chi+\kappa_{4 \alpha \beta}^{\prime} Q_{\alpha L} d_{\beta L}^{\prime c} \chi\right]+\text { H.c. }
$$

Defining the two column vectors

$$
\left(\psi_{d}^{+}\right)^{T}=\left(d_{1 L}^{c}, d_{2 L}^{c}, d_{3 L}^{c}, d_{2 L}^{\prime c}, d_{3 L}^{\prime c}\right), \quad\left(\psi_{d}^{-}\right)^{T}=\left(d_{1 L}, d_{2 L}, d_{3 L}, d_{2 L}^{\prime}, d_{3 L}^{\prime}\right),
$$

we can write mass term (90) in the form

$$
-\mathcal{L}=\left(\psi_{d}^{-}\right)^{T} X_{d} \psi_{d}^{+}+\text {H.c. }
$$


with

$$
X_{d}=\frac{1}{3 \sqrt{2}}\left(\begin{array}{ccccc}
\kappa_{21} v^{\prime} & \kappa_{22} v^{\prime} & \kappa_{23} v^{\prime} & \kappa_{22}^{\prime} v^{\prime} & \kappa_{23}^{\prime} v^{\prime} \\
\kappa_{421} u & \kappa_{422} u & \kappa_{423} u & \kappa_{422}^{\prime} u & \kappa_{423}^{\prime} u \\
\kappa_{431} u & \kappa_{432} u & \kappa_{433} u & \kappa_{432}^{\prime} u & \kappa_{433}^{\prime} u \\
\kappa_{421} w & \kappa_{422} w & \kappa_{423} w & \kappa_{422}^{\prime} w & \kappa_{423}^{\prime} w \\
\kappa_{431} w & \kappa_{432} w & \kappa_{433} w & \kappa_{432}^{\prime} w & \kappa_{433}^{\prime} w
\end{array}\right) .
$$

In the mass matrix (93), the second and the fourth rows are proportional; the third and the last rows are proportional too. Therefore, this matrix contains two massless particles which are the same as in Ref. [11].

The masslessness of one up-quark and two down-quarks calls for radiative corrections. Oneloop contributions can be obtained similarly to [11]. We can therefore check that the quarks get consistent masses at this level.

\section{Higgs sector}

As mentioned above, in Eqs. (34) and (39), the supersymmetric Higgs potential can be written as

$$
\begin{aligned}
V_{\text {susy eco }} \equiv & V_{\text {scalar }}+V_{\text {soft }} \\
= & \frac{\mu_{\chi}^{2}}{4}\left(|\chi|^{2}+\left|\chi^{\prime}\right|^{2}\right)+\frac{\mu_{\rho}^{2}}{4}\left(|\rho|^{2}+\left|\rho^{\prime}\right|^{2}\right) \\
& +\frac{g^{\prime 2}}{12}\left(-\frac{1}{3} \chi^{\dagger} \chi+\frac{1}{3} \chi^{\prime \dagger} \chi^{\prime}+\frac{2}{3} \rho^{\dagger} \rho-\frac{2}{3} \rho^{\prime \dagger} \rho^{\prime}\right)^{2} \\
& +\frac{g^{2}}{8}\left(\chi_{i}^{\dagger} \lambda_{i j}^{b} \chi_{j}-\chi_{i}^{\prime \dagger} \lambda_{i j}^{* b} \chi_{j}^{\prime}+\rho_{i}^{\dagger} \lambda_{i j}^{b} \rho_{j}-\rho_{i}^{\prime \dagger} \lambda_{i j}^{* b} \rho_{j}^{\prime}\right)^{2} \\
& +m_{\rho}^{2} \rho^{\dagger} \rho+m_{\chi}^{2} \chi^{\dagger} \chi+m_{\rho^{\prime}}^{2} \rho^{\prime \dagger} \rho^{\prime}+m_{\chi^{\prime}}^{2} \chi^{\prime \dagger} \chi^{\prime} .
\end{aligned}
$$

To look for mass spectrum of Higgs fields, we have to expand them around the VEVs as

$$
\begin{array}{ll}
\chi^{T}=\left(\frac{u+S_{1}+i A_{1}}{\sqrt{2}}, \chi^{-}, \frac{w+S_{2}+i A_{2}}{\sqrt{2}}\right), & \rho^{T}=\left(\rho_{1}^{+}, \frac{v+S_{5}+i A_{5}}{\sqrt{2}}, \rho_{2}^{+}\right), \\
\chi^{\prime T}=\left(\frac{u^{\prime}+S_{3}+i A_{3}}{\sqrt{2}}, \chi^{\prime+}, \frac{w^{\prime}+S_{4}+i A_{4}}{\sqrt{2}}\right), & \rho^{\prime T}=\left(\rho_{1}^{\prime-}, \frac{v^{\prime}+S_{6}+i A_{6}}{\sqrt{2}}, \rho_{2}^{\prime-}\right) .
\end{array}
$$

For the sake of simplicity, here we assume that the VEVs $u, u^{\prime}, v, v^{\prime}, w$ and $w^{\prime}$ are real. This means that the $\mathrm{CP}$ violation through the scalar exchanges is not considered in this work.

Returning to Eq. (94), by requirement of vanishing the linear terms in fields, we get, at the tree level approximation, the following constraint equations

$$
\begin{aligned}
& \mu_{\chi}^{2}+2 m_{\chi}^{2}=-\frac{g^{\prime 2}}{54}\left[w^{2}-w^{\prime 2}+u^{2}-u^{\prime 2}+2\left(v^{\prime 2}-v^{2}\right)\right] \\
&-\frac{g^{2}}{6}\left[2\left(u^{2}-u^{\prime 2}+w^{2}-w^{\prime 2}\right)+v^{\prime 2}-v^{2}\right], \\
& \mu_{\rho}^{2}+2 m_{\rho}^{2}=-\frac{2 g^{2 \prime}+9 g^{2}}{54}\left[2\left(v^{2}-v^{2 \prime}\right)+w^{2 \prime}-w^{2}+u^{\prime 2}-u^{2}\right], \\
& m_{\chi}^{2}+m_{\chi^{\prime}}^{2}+\mu_{\chi}^{2}=0,
\end{aligned}
$$




$$
\begin{aligned}
& m_{\rho}^{2}+m_{\rho \prime}^{2}+\mu_{\rho}^{2}=0, \\
& \left(w^{2}-u^{2}\right) u^{\prime} w^{\prime}=\left(w^{\prime 2}-u^{\prime 2}\right) u w .
\end{aligned}
$$

It is noteworthy that Eq. (100) implies the matching condition previously mentioned in (42). Consequently, the model contains a pair of Higgs triplet $\chi$ and antitriplet $\chi^{\prime}$ with the VEVs in top and bottom elements governed by the relation: $u / w=u^{\prime} / w^{\prime}$.

The squared-mass matrix derived from $(94)$ can be divided into $(6 \times 6)$ matrices respective to the charged, scalar and pseudoscalar bosons. Note that there is no mixing among the scalar and pseudoscalar bosons. We consider, first, in the case of the pseudoscalar bosons. There are two massless particles, namely, $A_{5}, A_{6}$. Four others are mixing in the base of $\left(A_{1}, A_{3}, A_{2}, A_{4}\right)$, their $(4 \times 4)$ squared-mass matrix takes the form:

$$
M_{4 A}^{2}=-\frac{g^{2}}{4}\left(\begin{array}{cccc}
-w^{\prime 2} & -w^{\prime} w & u^{\prime} w^{\prime} & u^{\prime} w \\
& -w^{2} & u w^{\prime} & u w \\
& & -u^{2 \prime} & -u u^{\prime} \\
& & & -u^{2}
\end{array}\right)
$$

To obtain eigenvalues and eigenstates, we change the basis to such $\left(A_{1}^{\prime}, A_{2}^{\prime}, A_{3}^{\prime}, A_{4}^{\prime}\right)$ as

$$
\begin{array}{ll}
A_{1}^{\prime}=s_{\beta} A_{1}-c_{\beta} A_{3}, & A_{3}^{\prime}=c_{\beta} A_{1}+s_{\beta} A_{3}, \\
A_{2}^{\prime}=s_{\beta} A_{2}-c_{\beta} A_{4}, & A_{4}^{\prime}=c_{\beta} A_{2}+s_{\beta} A_{4},
\end{array}
$$

where

$$
t_{\beta} \equiv \frac{w}{w^{\prime}}
$$

Combining this with the relation (42), we have also

$$
t_{\beta}=\frac{w}{w^{\prime}}=\frac{u}{u^{\prime}} .
$$

In the new basis $\left(A_{1}^{\prime}, A_{3}^{\prime}, A_{2}^{\prime}, A_{4}^{\prime}\right)$, the squared-mass matrix (101) can be rewritten as

$$
M_{4 A^{\prime}}^{2}=-\frac{g^{2}}{4}\left(\begin{array}{cccc}
0 & 0 & 0 & 0 \\
0 & -w^{2}-w^{\prime 2} & 0 & w u+w^{\prime} u^{\prime} \\
0 & 0 & 0 & 0 \\
0 & w u+w^{\prime} u^{\prime} & 0 & -u^{2}-u^{\prime 2}
\end{array}\right)
$$

We see that $A_{1}^{\prime}$ and $A_{2}^{\prime}$ are Goldstone bosons, whereas the remaining states $A_{3}^{\prime}$ and $A_{4}^{\prime}$ are mixing. Diagonalizing the later, we obtain another Goldstone boson $\varphi_{A}$ and one massive state $\phi_{A}$

$$
\varphi_{A}=s_{\theta} A_{3}^{\prime}+c_{\theta} A_{4}^{\prime}, \quad \phi_{A}=c_{\theta} A_{3}^{\prime}-s_{\theta} A_{4}^{\prime} .
$$

The mass of $\phi_{A}$ is given by

$$
m_{\phi_{A}}^{2}=\frac{g^{2}}{4}\left(1+t_{\theta}^{2}\right)\left(w^{2}+w^{\prime 2}\right)=m_{X}^{2} .
$$

The above equation shows that the Higgs and gauge bosons have the same mass. 
Now we turn to the scalar sector. In this sector, six particles are mixing in terms of an $6 \times 6$ squared-mass matrix. In the base of $\left(S_{1}, S_{2}, S_{3}, S_{4}, S_{5}, S_{6}\right)$, this matrix is given by

$$
M_{6 S}^{2}=\frac{1}{2}\left(\begin{array}{cccccc}
m_{S 11}^{2} & m_{S 12}^{2} & m_{S 13}^{2} & m_{S 14}^{2} & m_{S 15}^{2} & m_{S 16}^{2} \\
& m_{S 22}^{2} & m_{S 23}^{2} & m_{S 24}^{2} & m_{S 25}^{2} & m_{S 26}^{2} \\
& & m_{S 33}^{2} & m_{S 34}^{2} & m_{S 35}^{2} & m_{S 36}^{2} \\
& & & m_{S 44}^{2} & m_{S 45}^{2} & m_{S 46}^{2} \\
& & & & m_{S 55}^{2} & m_{S 56}^{2} \\
& & & & & m_{S 66}^{2}
\end{array}\right),
$$

where the matrix elements are given in Appendix A.

To study physical eigenvalues and eigenstates of (108), we change the basis to such $\left(S_{1}^{\prime}, S_{2}^{\prime}, S_{3}^{\prime}, S_{4}^{\prime}, S_{5}^{\prime}, S_{6}^{\prime}\right)$ as

$$
\left(\begin{array}{c}
S_{1} \\
S_{2} \\
S_{3} \\
S_{4} \\
S_{5} \\
S_{6}
\end{array}\right)=\left(\begin{array}{cccccc}
s_{\theta} & -c_{\theta} & 0 & 0 & 0 & 0 \\
c_{\theta} & s_{\theta} & 0 & 0 & 0 & 0 \\
0 & 0 & s_{\theta} & -c_{\theta} & 0 & 0 \\
0 & 0 & c_{\theta} & s_{\theta} & 0 & 0 \\
0 & 0 & 0 & 0 & \frac{v^{\prime}}{\sqrt{v^{2}+v^{\prime 2}}} & \frac{-v}{\sqrt{v^{2}+v^{\prime 2}}} \\
0 & 0 & 0 & 0 & \frac{v}{\sqrt{v^{2}+v^{\prime 2}}} & \frac{v^{\prime}}{\sqrt{v^{2}+v^{\prime 2}}}
\end{array}\right)\left(\begin{array}{c}
S_{1}^{\prime} \\
S_{2}^{\prime} \\
S_{3}^{\prime} \\
S_{4}^{\prime} \\
S_{5}^{\prime} \\
S_{6}^{\prime}
\end{array}\right) .
$$

In the new basis $\left(S_{1}^{\prime}, S_{3}^{\prime}, S_{6}^{\prime}, S_{2}^{\prime}, S_{4}^{\prime}, S_{5}^{\prime}\right)$, the matrix (108) becomes

$$
M_{6 S^{\prime}}^{2}=\left(\begin{array}{ccc}
M_{3 S^{\prime}}^{2} & 0 & 0 \\
0 & M_{2 S^{\prime}}^{2} & 0 \\
0 & 0 & 0
\end{array}\right) .
$$

We see that the mass spectrum contains one massless particle $S_{5}^{\prime}$. The submatrices of $\left(S_{2}^{\prime}, S_{4}^{\prime}\right)$ and $\left(S_{1}^{\prime}, S_{3}^{\prime}, S_{6}^{\prime}\right)$ are decoupled and, respectively, given by

$$
\begin{aligned}
& M_{2 S^{\prime}}^{2}=\frac{g^{2}}{4}\left(1+t_{\theta}^{2}\right)\left(\begin{array}{cc}
w^{\prime 2} & -w w^{\prime} \\
-w w^{\prime} & w^{2}
\end{array}\right), \\
& M_{3 S^{\prime}}^{2}=\left(\begin{array}{ccc}
\frac{18 g^{2}+g^{\prime 2}}{54 c_{\theta}^{2}} w^{2} & -\frac{18 g^{2}+g^{\prime 2}}{54 c_{\theta}^{2}} w w^{\prime} & \frac{g^{2}\left(9 g^{2}+2 g^{\prime 2}\right)}{54 c_{\theta}} \sqrt{v^{2}+v^{\prime 2}} w \\
& \frac{18 g^{2}+g^{\prime 2}}{54 c_{\theta}^{2}} w^{\prime 2} & -\frac{g^{2}\left(9 g^{2}+2 g^{\prime 2}\right)}{54 c_{\theta}} \sqrt{v^{2}+v^{\prime 2}} w^{\prime} \\
& & \frac{9 g^{2}+2 g^{\prime 2}}{27}\left(v^{2}+v^{\prime 2}\right)
\end{array}\right) .
\end{aligned}
$$

The matrix (111) gives us one massless field

$$
\varphi_{S_{24}}=s_{\beta} S_{2}^{\prime}+c_{\beta} S_{4}^{\prime},
$$

and another massive

$$
\phi_{S_{24}}=c_{\beta} S_{2}^{\prime}-s_{\beta} S_{4}^{\prime}
$$

with the mass:

$$
m_{\phi_{S_{24}}}^{2}=\frac{g^{2}}{4}\left(1+t_{\theta}^{2}\right)\left(w^{2}+w^{\prime 2}\right)=m_{X}^{2} .
$$


Let us note that $\phi_{A}$ and $\phi_{S_{24}}$ have the same mass, which can be combined to become a physical neutral complex field $H_{X}^{0}=\left(\phi_{S_{24}}+i \phi_{A}\right) / \sqrt{2}$ with mass equal to $m_{X}$ of the neutral nonHermitian gauge boson $X^{0}$.

To obtain the physical fields in $M_{3 S^{\prime}}^{2}$, we use the following transformation:

$$
\left(\begin{array}{l}
S_{1 a}^{\prime} \\
S_{3 a}^{\prime} \\
S_{6 a}^{\prime}
\end{array}\right)=\left(\begin{array}{ccc}
c_{\beta} & s_{\beta} & 0 \\
-s_{\beta} & c_{\beta} & 0 \\
0 & 0 & 1
\end{array}\right)\left(\begin{array}{c}
S_{1}^{\prime} \\
S_{3}^{\prime} \\
S_{6}^{\prime}
\end{array}\right) .
$$

In the new basis $\left(S_{1 a}^{\prime}, S_{3 a}^{\prime}, S_{6 a}^{\prime}\right)$, the matrix (112) becomes

$$
M_{a 3 S^{\prime}}^{2}=\left(\begin{array}{ccc}
0 & 0 & 0 \\
0 & m_{33 a}^{2} & -m_{36 a}^{2} \\
0 & -m_{36 a}^{2} & m_{66 a}^{2}
\end{array}\right),
$$

where

$$
\begin{aligned}
& m_{33 a}^{2}=\frac{18 g^{2}+g^{\prime 2}}{54 c_{\theta}^{2}}\left(w^{2}+w^{\prime 2}\right), \quad m_{66 a}^{2}=\frac{9 g^{2}+2 g^{\prime 2}}{27}\left(v^{2}+v^{\prime 2}\right), \\
& m_{36 a}^{2}=\frac{g^{2}\left(9 g^{2}+2 g^{\prime 2}\right) \sqrt{\left(v^{2}+v^{\prime 2}\right)\left(w^{2}+w^{\prime 2}\right)}}{54 c_{\theta}} .
\end{aligned}
$$

The field $S_{1 a}^{\prime}$ is physical and massless. The submatrix of $\left(S_{3 a}^{\prime}, S_{6 a}^{\prime}\right)$ is decoupled, and therefore the diagonalization yields the eigenvalues

$$
\begin{aligned}
& m_{\varphi_{S_{a 36}}}^{2}=\frac{1}{2}\left[m_{33 a}^{2}+m_{66 a}^{2}-\sqrt{\left(m_{33 a}^{2}-m_{66 a}^{2}\right)^{2}+4 m_{36 a}^{4}}\right] \\
& m_{\phi_{S_{a 36}}}^{2}=\frac{1}{2}\left[m_{33 a}^{2}+m_{66 a}^{2}+\sqrt{\left(m_{33 a}^{2}-m_{66 a}^{2}\right)^{2}+4 m_{36 a}^{4}}\right]
\end{aligned}
$$

with the respective eigenstates

$$
\varphi_{S_{a 36}}=s_{\alpha} S_{3}^{\prime}+c_{\alpha} S_{6}^{\prime}, \quad \phi_{S_{a 36}}=c_{\alpha} S_{3}^{\prime}-s_{\alpha} S_{6}^{\prime},
$$

where

$$
t_{2 \alpha} \equiv \frac{-2 m_{36 a}^{2}}{m_{66 a}^{2}-m_{33 a}^{2}} .
$$

Finally, we consider the mass spectrum of charged Higgs bosons. In the base of $\left(\chi^{+}, \chi^{+\prime}\right.$, $\left.\rho_{1}^{+}, \rho_{2}^{+}, \rho_{1}^{+\prime}, \rho_{2}^{+\prime}\right)$, the squared-mass matrix can be written as

$$
M_{6 \text { charged }}^{2}=\frac{g^{2}}{4}\left(\begin{array}{cccccc}
m_{\chi^{-} \chi^{+}}^{2} & m_{\chi^{-} \chi^{+\prime}}^{2} & u v & v w & -u v^{\prime} & -v^{\prime} w \\
& m_{\chi^{-1} \chi^{+\prime}}^{2} & -v u^{\prime} & -w^{\prime} v & v^{\prime} u^{\prime} & v^{\prime} w^{\prime} \\
& & m_{\rho_{1}^{-} \rho_{1}^{+}}^{2} & m_{\rho_{1}^{-} \rho_{2}^{+}}^{2} & -v v^{\prime} & 0 \\
& & -m_{\rho_{2}^{-} \rho_{2}^{+}}^{2} & 0 & -v v^{\prime} \\
& & & m_{\rho_{1}^{-1} \rho_{1}^{+\prime}}^{2} & m_{\rho_{1}^{-1} \rho_{2}^{+\prime}}^{2} \\
& & & & m_{\rho_{2}^{-1} \rho_{2}^{+\prime}}^{2}
\end{array}\right) \text {, }
$$


where

$$
\begin{aligned}
& m_{\chi^{-} \chi^{+}}^{2}=w^{2 \prime}+u^{2 \prime}+\left(v^{2}-v^{2 \prime}\right), \quad m_{\chi^{-} \chi^{+\prime}}^{2}=-w w^{\prime}-u u^{\prime}, \\
& m_{\rho_{1}^{-} \rho_{2}^{+}}^{2}=u^{2}-u^{2 \prime}+v^{2 \prime}, \quad m_{\chi^{-\prime} \chi^{+\prime}}^{2}=u^{2}+w^{2}-\left(v^{2}-v^{2 \prime}\right), \\
& m_{\rho_{1}^{-} \rho_{2}^{+}}^{2}=u w-u^{\prime} w^{\prime}, \quad m_{\rho_{1}^{\prime} \rho_{1}^{\prime+}}^{2}=u^{\prime 2}-u^{2}+v^{2}, \quad m_{\rho_{1}^{-\prime} \rho_{2}^{+\prime}}^{2}=-m_{\rho_{1}^{-} \rho_{2}^{+}}^{2}, \\
& m_{\rho_{2}^{-} \rho_{2}^{+}}^{2}=w^{2}-w^{2 \prime}+v^{2 \prime}, \quad m_{\rho_{2}^{-\prime} \rho_{2}^{+\prime}}^{2}=w^{2 \prime}-w^{2}+v^{2} .
\end{aligned}
$$

To diagonalize the matrix (121), we choose a new basis as follows

$$
\left(\begin{array}{c}
\chi_{a}^{+} \\
\chi_{a}^{\prime+}
\end{array}\right)=O\left(\begin{array}{c}
\chi^{+} \\
\chi^{\prime+}
\end{array}\right), \quad\left(\begin{array}{c}
\rho_{1 a}^{+} \\
\rho_{2 a}^{+}
\end{array}\right)=O_{1}\left(\begin{array}{c}
\rho_{1}^{+} \\
\rho_{2}^{+}
\end{array}\right), \quad\left(\begin{array}{c}
\rho_{1 a}^{\prime+} \\
\rho_{2 a}^{\prime+}
\end{array}\right)=O_{1}\left(\begin{array}{c}
\rho_{1}^{\prime+} \\
\rho_{2}^{\prime+}
\end{array}\right),
$$

with

$$
O \equiv\left(\begin{array}{cc}
s_{\beta} & -c_{\beta} \\
c_{\beta} & s_{\beta}
\end{array}\right), \quad O_{1} \equiv\left(\begin{array}{cc}
-c_{\theta} & s_{\theta} \\
s_{\theta} & c_{\theta}
\end{array}\right)
$$

In the base of $\left(\chi_{a}^{+}, \chi_{a}^{\prime+}, \rho_{1 a}^{+}, \rho_{2 a}^{+}, \rho_{1 a}^{\prime+}, \rho_{2 a}^{+}\right)$, the matrix (121) becomes

$$
M_{a 6 \text { charged }}^{2}=\frac{g^{2}}{4}\left(\begin{array}{cccccc}
m_{a 11}^{2} & m_{a 12}^{2} & 0 & m_{a 14}^{2} & 0 & m_{a 16}^{2} \\
& m_{a 22}^{2} & 0 & 0 & 0 & 0 \\
& & v^{\prime 2} & 0 & -v v^{\prime} & 0 \\
& & & m_{a 44}^{2} & 0 & -v v^{\prime} \\
& & & & v^{2} & 0 \\
& & & & & m_{a 66}^{2}
\end{array}\right) \text {, }
$$

where

$$
\begin{aligned}
& m_{a 11}^{2}=-c_{2 \beta}\left(t_{\gamma}^{2}-1\right) v^{\prime 2}, \quad m_{a 12}^{2}=-s_{2 \beta}\left(t_{\gamma}^{2}-1\right) v^{\prime 2}, \\
& m_{a 22}^{2}=c_{2 \beta}\left(t_{\gamma}^{2}-1\right) v^{\prime 2}+\left(1+t_{\beta}^{2}\right)\left(1+t_{\theta}^{2}\right) w^{\prime 2}, \\
& m_{a 14}^{2}=\sqrt{\left(1+t_{\beta}^{2}\right)\left(1+t_{\theta}^{2}\right)} w^{\prime} v, \quad m_{a 16}^{2}=\sqrt{\left(1+t_{\beta}^{2}\right)\left(1+t_{\theta}^{2}\right)} w^{\prime} v^{\prime}, \\
& m_{a 44}^{2}=\left(t_{\beta}^{2}-1\right)\left(t_{\theta}^{2}+1\right) w^{\prime 2}+v^{\prime 2}, \\
& m_{a 66}^{2}=-\left(t_{\beta}^{2}-1\right)\left(t_{\theta}^{2}+1\right) w^{\prime 2}+v^{2}, \quad t_{\gamma} \equiv \frac{v}{v^{\prime}} .
\end{aligned}
$$

Since the block intersected by the third, fifth rows and columns is decoupled, it can be diagonalized and this yields two eigenvalues as follows

$$
\begin{aligned}
& m_{\varrho_{1}^{+}}^{2}=\frac{g^{2}}{4}\left(v^{2}+v^{\prime 2}\right)=m_{W}^{2}, \\
& m_{\varrho_{2}^{+}}^{2}=0 .
\end{aligned}
$$

Here the Goldstone boson $\varrho_{2}^{+}$and Higgs boson $\varrho_{1}^{+}$are, respectively, defined by 


$$
\varrho_{1}^{+}=c_{\gamma} \rho_{1 a}^{+}-s_{\gamma} \rho_{1 a}^{\prime+}, \quad \varrho_{2}^{+}=s_{\gamma} \rho_{1 a}^{+}+c_{\gamma} \rho_{1 a}^{\prime+} .
$$

Eq. (126) shows that one charged Higgs boson has the mass equal to those of $W$ boson, i.e. $m_{\varrho_{1}^{ \pm}}=m_{W^{ \pm}}$, this result is in agreement with the experimental current limit $m_{H^{ \pm}}>79.3 \mathrm{GeV}$, $\mathrm{CL}=95 \%$ [24].

The remaining part of $\left(\chi_{a}^{+}, \chi_{a}^{\prime+}, \rho_{2 a}^{+}, \rho_{2 a}^{\prime+}\right)$ is still mixing in terms of an $4 \times 4$ submatrix of (124). Under the constraints (15) we will split this matrix into two terms,

$$
M_{a 4 \text { charged }}^{2}=M_{b 4 \text { charged }}^{2}+M_{c 4 \text { charged }}^{2},
$$

satisfying the condition: $\left|M_{c 4 \text { charged }}^{2}\right| \ll\left|M_{b 4 \text { charged }}^{2}\right|$. In this case, the first matrix chosen consists of elements of the leading-order terms in $w^{2}$ or $w^{\prime 2}$ of $M_{a 4 \text { charged }}^{2}$, while $M_{c 4 \text { charged }}^{2}$ contains the remaining terms. The matrix $M_{a 4 \text { charged }}^{2}$ can therefore be diagonalized with the contribution of $M_{c 4 \text { charged }}^{2}$ considered as a perturbation. The eigenvalues and eigenstates are, respectively, obtained up to the first order contributions as follows

$$
\begin{array}{ll}
m_{\zeta_{1}^{+}}^{2}=\frac{g^{2}}{4} m_{a 11}^{2}, & \zeta_{1}^{+}=\chi_{a}^{+}-\frac{m_{a 12}^{2}}{\tilde{m}_{\zeta_{2}^{+}}^{2}} \chi_{a}^{\prime+}-\frac{m_{a 14}^{2}}{\tilde{m}_{\zeta_{3}^{+}}^{2}} \rho_{a 2}^{+}-\frac{m_{a 16}^{2}}{\tilde{m}_{\zeta_{4}^{+}}^{2}} \rho_{a 2}^{\prime+}, \\
m_{\zeta_{2}^{+}}^{2}=\frac{g^{2}}{4} m_{a 22}^{2}, & \zeta_{2}^{+}=\chi_{a}^{\prime+}+\frac{m_{a 12}^{2}}{\tilde{m}_{\zeta_{2}^{+}}^{2}} \chi_{a}^{+}, \\
m_{\zeta_{3}^{+}}^{2}=\frac{g^{2}}{4} m_{a 44}^{2}, & \zeta_{3}^{+}=\rho_{2 a}^{+}+\frac{m_{a 14}^{2}}{\tilde{m}_{\zeta_{3}^{+}}^{2}} \chi_{a}^{+}-\frac{v v^{\prime}}{\tilde{m}_{\zeta_{3}^{+}}^{2}-\tilde{m}_{\zeta_{4}^{+}}^{2}} \rho_{a 2}^{\prime+}, \\
m_{\zeta_{4}^{+}}^{2}=\frac{g^{2}}{4} m_{a 66}^{2}, & \zeta_{4}^{+}=\rho_{2 a}^{\prime+}+\frac{m_{a 16}^{2}}{\tilde{m}_{\zeta_{3}^{+}}^{2}} \chi_{a}^{+}+\frac{v v^{\prime}}{\tilde{m}_{\zeta_{3}^{+}}^{2}-\tilde{m}_{\zeta_{4}^{+}}^{2}} \rho_{a 2}^{+} .
\end{array}
$$

Here we have denoted

$$
\tilde{m}_{\zeta_{2}^{+}}^{2}=\left(1+t_{\beta}^{2}\right)\left(1+t_{\theta}^{2}\right) w^{\prime 2}, \quad \tilde{m}_{\zeta_{3}^{+}}^{2}=-\tilde{m}_{\zeta_{4}^{+}}^{2}=\left(t_{\beta}^{2}-1\right)\left(t_{\theta}^{2}+1\right) w^{\prime 2}
$$

If we control parameters by the condition below

$$
\frac{v^{2}}{w^{\prime 2}}=\left(t_{\beta}^{2}-1\right)\left(t_{\theta}^{2}+1\right)
$$

then $m_{\zeta_{4}^{+}}^{2}=0$. Therefore, in the mass spectrum of charged Higgs, we have two zero eigenvalues which correspond to four Goldstone bosons, namely $\zeta_{4}^{ \pm}, \varrho_{2}^{ \pm}$.

Finally, let us summarize the physical fields of the scalar sector in the model. There are eight neutral massless particles: five pseudoscalars $A_{5}, A_{6}, A_{1}^{\prime}, A_{2}^{\prime}, \varphi_{A}$, and three scalars $S_{5}^{\prime}, \varphi_{S_{24}}, S_{1 a}^{\prime}$. There are one complex neutral Higgs $H_{X}^{0}$ with mass equal to the mass $m_{X}$ of the neutral nonHermitian gauge boson $X$, and two massive scalars $\varphi_{S_{a 36}}, \phi_{S_{a 36}}$. There are two charged massless scalar fields $\varrho_{2}^{ \pm}$and $\varrho_{4}^{ \pm}$, and four massive charged bosons $\varrho_{1}^{ \pm}, \zeta_{1}^{ \pm}, \zeta_{2}^{ \pm}, \zeta_{3}^{ \pm}$. The first charged Higgs boson has a mass equal to those of $W$ boson: $m_{\varrho_{1}^{ \pm}}=m_{W}$. 


\section{Conclusions}

In this paper, we have constructed a supersymmetric version of the economical 3-3-1 model of Refs. [8-10] which includes right-handed neutrinos with the minimal scalar sector. The Higgs sector was in detail studied: the eigenvalues and physical states were derived. The constraint equations and the gauge boson identification establish a relation between the vacuum expectation values at the top and bottom elements of the Higgs triplet $\chi$ and its supersymmetric counterpart $\chi^{\prime}$. Because of this relation, the exact diagonalization of neutral gauge boson sector has been performed. The gauge bosons and their associated Goldstone ones are mixing in the same way as in non-supersymmetric version. The matrix of neutral gauge boson mixing is separated into two parts and one of them is those in the non-supersymmetric version. There is similarity in the gauge boson mixing in both supersymmetric and non-supersymmetric versions. This is also correct in the case of gauginos.

The model contains a heavy neutral complex scalar with mass equal to those of the neutral non-Hermitian gauge boson $X^{0}$ and a charged scalar with mass equal to those of the $W$ boson in the Standard Model, i.e., $m_{H_{X}}=m_{X}, m_{\varrho_{1}^{+}}=m_{W}$. This value is in good agreement with the present bound [24] $m_{H^{ \pm}}>79.3 \mathrm{GeV}, \mathrm{CL}=95 \%$. We have also shown that the boson sector and the fermion sector get masses in the same way as in the non-supersymmetric case. We have shown that the usual quarks have masses proportional to VEVs of the neutral scalars which belong to doublets while the exotic quarks and new gauge bosons gain masses of order of VEVs of the scalar of the singlets of the Standard Model group. The usual quarks have masses proportional to VEVs of the neutral scalars which belong to doublets of the Standard Model $-v_{\rho}, v_{\chi_{1}}, v_{\rho^{\prime}}$ and $v_{\chi_{1}^{\prime}}$, while the exotic quarks and new gauge bosons gain masses of order of VEVs $v_{\chi_{2}}$ and $v_{\chi_{2}^{\prime}}$ of the scalars which break the 3-3-1 symmetry down to the Standard Model, in the other words, the scalar of the singlets of the above group. As in non-supersymmetric version, at the tree level, one up-quark and two down-quarks are massless. However, the one-loop correction will give all of them consistent masses.

It is known that the economical (non-supersymmetric) 3-3-1 model does not furnish the candidate for self-interacting dark matter. With a larger content of the scalar sector, the supersymmetric version is expected to have the candidate for SIDM. The preliminary analysis leads us to conclusion that one neutral scalar contains the properties of SIDM like stability, neutrality and the Universe's overpopulationless. Hence, in contrast with the non-supersymmetric version, the considered model contains the scalar satisfying the properties of SIDM. We will return to this topic in the future study.

Due to the minimal content of the scalar sector, the significant number of free parameters is reduced. In this model, the lepton number violation exists in the neutrino and exotic quark sectors. Certainly, the model contains very rich phenomenology and it deserves further studies.

\section{Acknowledgements}

D.T.H. is grateful to Aichi University of Education, Kariya, Japan for supporting this work. H.N.L. would like to thank Professor R. Ruffini and Members of ICRANet in Pescara for kind help and hospitality during his visit where this work was initialed. The work was also supported in part by National Council for Natural Sciences of Vietnam under grant No. 410604. M.C.R. is grateful to Conselho Nacional de Desenvolvimento Científico e Tecnológico (CNPq) under the processes 309564/2006-9 for supporting his work. 


\section{Appendix A. The mass matrix elements for scalar neutral Higgs bosons}

$$
\begin{aligned}
& m_{S 11}^{2}=\frac{g^{2}}{2} w^{\prime 2}+\frac{1}{27}\left(18 g^{2}+g^{\prime 2}\right) u^{2}, \quad m_{S 12}^{2}=-\frac{g^{2}}{2} u^{\prime} w^{\prime}+\frac{1}{27}\left(18 g^{2}+g^{2 \prime}\right) u w \\
& m_{S 13}^{2}=-\frac{g^{2}}{2} w w^{\prime}-\frac{1}{27}\left(18 g^{2}+g^{2 \prime}\right) u u^{\prime}, \quad m_{S 14}^{2}=\frac{g^{2}}{2} u^{\prime} w-\frac{1}{27}\left(18 g^{2}+g^{2 \prime}\right) u w^{\prime}, \\
& m_{S 15}^{2}=-\frac{g^{2}}{27}\left(2 g^{2 \prime}+9 g^{2}\right) u v, \quad m_{S 16}^{2}=\frac{g^{2}}{27}\left(2 g^{2 \prime}+9 g^{2}\right) u v^{\prime}, \\
& m_{S 22}^{2}=\frac{g^{2}}{2} u^{\prime 2}+\frac{1}{27}\left(18 g^{2}+g^{2 \prime}\right) w^{2}, \quad m_{S 23}^{2}=\frac{g^{2}}{2} u^{\prime} w-\frac{1}{27}\left(18 g^{2}+g^{2 \prime}\right) u^{\prime} w, \\
& m_{S 24}^{2}=-\frac{g^{2}}{2} u u^{\prime}-\frac{1}{27}\left(18 g^{2}+g^{2 \prime}\right) w w^{\prime}, \quad m_{S 25}^{2}=-\frac{g^{2}}{27}\left(9 g^{2}+2 g^{2 \prime}\right) w v, \\
& m_{S 26}^{2}=\frac{g^{2}}{27}\left(2 g^{2 \prime}+9 g^{2}\right) w v^{\prime}, \quad m_{S 33}^{2}=\frac{1}{27}\left(g^{2 \prime}+18 g^{2}\right) u^{\prime 2}+\frac{g^{2}}{2} w^{2}, \\
& m_{S 34}^{2}=\frac{1}{27}\left(g^{2 \prime}+18 g^{2}\right) u^{\prime} w^{\prime}-\frac{g^{2}}{2} u w, \quad m_{S 35}^{2}=\frac{g^{2}}{27}\left(2 g^{2 \prime}+9 g^{2}\right) u^{\prime} v, \\
& m_{36}^{2}=-\frac{g^{2}}{27}\left(2 g^{2 \prime}+9 g^{2}\right) u^{\prime} v^{\prime}, \quad m_{S 44}^{2}=\frac{1}{27}\left(g^{2 \prime}+18 g^{2}\right) w^{\prime 2}+\frac{g^{2}}{2} u^{2}, \\
& m_{S 45}^{2}=\frac{g^{2}}{27}\left(2 g^{2 \prime}+9 g^{2}\right) v w^{\prime}, \quad m_{46}^{2}=-\frac{g^{2}}{27}\left(2 g^{2 \prime}+9 g^{2}\right) w^{\prime} v^{\prime}, \\
& m_{S 55}^{2}=\frac{2}{27}\left(2 g^{2 \prime}+9 g^{2}\right) v^{2}, \quad m_{S 56}^{2}=-\frac{2}{27}\left(2 g^{2 \prime}+9 g^{2}\right) v v^{\prime}, \\
& m_{S 66}^{2}=\frac{2}{27}\left(2 g^{2 \prime}+9 g^{2}\right) v^{\prime 2} . \quad
\end{aligned}
$$

\section{References}

[1] SuperKamiokande Collaboration, Y. Fukuda, et al., Phys. Rev. Lett. 81 (1998) 1158; SuperKamiokande Collaboration, Y. Fukuda, et al., Phys. Rev. Lett. 81 (1998) 1562; SuperKamiokande Collaboration, Y. Fukuda, et al., Phys. Rev. Lett. 82 (1999) 2644; SuperKamiokande Collaboration, Y. Fukuda, et al., Phys. Rev. Lett. 85 (2000) 3999; Y. Suzuki, Nucl. Phys. B (Proc. Suppl.) 77 (1999) 35;

S. Fukuda, et al., Phys. Rev. Lett. 86 (2001) 5651;

Y. Ashie, et al., Phys. Rev. Lett. 93 (2004) 101801.

[2] KamLAND Collaboration, K. Eguchi, et al., Phys. Rev. Lett. 90 (2003) 021802; T. Araki, et al., Phys. Rev. Lett. 94 (2005) 081801.

[3] SNO Collaboration, Q.R. Ahmad, et al., Phys. Rev. Lett. 89 (2002) 011301; SNO Collaboration, Q.R. Ahmad, et al., Phys. Rev. Lett. 89 (2002) 011302; SNO Collaboration, Q.R. Ahmad, et al., Phys. Rev. Lett. 92 (2004) 181301;

B. Aharmim, et al., Phys. Rev. C 72 (2005) 055502.

[4] F. Pisano, V. Pleitez, Phys. Rev. D 46 (1992) 410;

P.H. Frampton, Phys. Rev. Lett. 69 (1992) 2889;

R. Foot, et al., Phys. Rev. D 47 (1993) 4158.

[5] M. Singer, et al., Phys. Rev. D 22 (1980) 738.

[6] R. Foot, H.N. Long, T.A. Tran, Phys. Rev. D 50 (1994) R34; J.C. Montero, et al., Phys. Rev. D 47 (1993) 2918; 
H.N. Long, Phys. Rev. D 54 (1996) 4691;

H.N. Long, Phys. Rev. D 53 (1996) 437.

[7] J.C. Montero, V. Pleitez, M.C. Rodriguez, Phys. Rev. D 70 (2004) 075004.

[8] W.A. Ponce, Y. Giraldo, L.A. Sanchez, Phys. Rev. D 67 (2003) 075001.

[9] P.V. Dong, H.N. Long, D.T. Nhung, D.V. Soa, Phys. Rev. D 73 (2006) 035004.

[10] P.V. Dong, H.N. Long, D.V. Soa, Phys. Rev. D 73 (2006) 075005.

[11] P.V. Dong, Tr.T. Huong, D.T. Huong, H.N. Long, Phys. Rev. D 74 (2006) 053003.

[12] P.V. Dong, H.N. Long, D.V. Soa, Neutrino masses in the economical 3-3-1 model, hep-ph/0610381.

[13] J. Wess, J. Bagger, Supersymmetry and Supergravity, second ed., Princeton Univ. Press, Princeton, NJ, 1992; H.E. Haber, G.L. Kane, Phys. Rep. 117 (1985) 75.

[14] D.T. Huong, M.C. Rodriguez, H.N. Long, Scalar sector of supersymmetric SU(3) $C \otimes \operatorname{SU}(3)_{L} \otimes \mathrm{U}_{(1)_{N}}$ model with right-handed neutrinos, hep-ph/0508045.

[15] P.V. Dong, D.T. Huong, M.C. Rodriguez, H.N. Long, Eur. Phys. J. C 48 (2006) 229, hep-ph/0604028.

[16] H.N. Long, P.B. Pal, Mod. Phys. Lett. A 13 (1998) 2355.

[17] D. Fregolente, M.D. Tonasse, Phys. Lett. B 555 (2003) 7; H.N. Long, N.Q. Lan, Europhys. Lett. 64 (2003) 571.

[18] V. Silveira, A. Zee, Phys. Lett. B 161 (1985) 136; D.E. Holz, A. Zee, Phys. Lett. B 517 (2000) 239;

C.P. Burgess, M. Pospelov, T. ter Veldhuis, Nucl. Phys. B 619 (2002) 709;

B.C. Bento, O. Bertolami, R. Rosenfeld, L. Teodoro, Phys. Rev. D 62 (2000) 041302;

J. McDonald, Phys. Rev. Lett. 88 (2002) 091304.

[19] D.N. Spergel, P.J. Steinhardt, Phys. Rev. Lett. 84 (2000) 3760.

[20] L. Girardello, M.T. Grisaru, Nucl. Phys. B 194 (1982) 65.

[21] P.V. Dong, H.N. Long, Eur. Phys. J. C 42 (2005) 325.

[22] D.A. Gutierrez, W.A. Ponce, L.A. Sanchez, Int. J. Mod. Phys. A 21 (2006) 2217.

[23] D. Chang, H.N. Long, Phys. Rev. D 73 (2006) 053006.

[24] W.-M. Yao, et al., Particle Data Group, J. Phys. G: Nucl. Part. Phys. 33 (1) (2006) 32. 